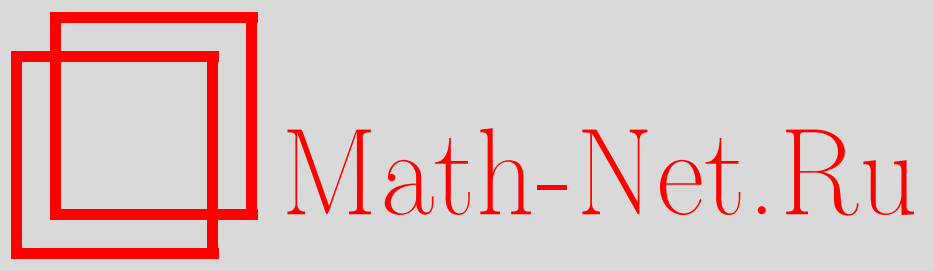

В. В. Пупышев, Двумерное кулоновское рассеяние квантовой частицы: низкоэнергетические асимптотики, TMФ, 2016, том 188, номер 1, 49-75

DOI: https://doi.org/10.4213/tmf9026

Использование Общероссийского математического портала Math-Net.Ru подразумевает, что вы прочитали и согласны с пользовательским соглашением http://www . mathnet.ru/rus/agreement

Параметры загрузки:

IP: 54.198 .64 .247

26 апреля 2023 г., $11: 35: 22$

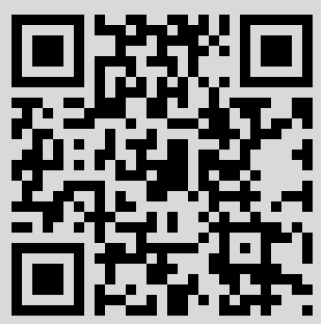




\title{
ФИЗИКА
}

Том 188, № 1

июль, 2016

\section{ДВУМЕРНОЕ КУЛОНОВСКОЕ РАССЕЯНИЕ КВАНТОВОЙ ЧАСТИЦЫ: НИЗКОЭНЕРГЕТИЧЕСКИЕ АСИМПТОТИКИ}

\begin{abstract}
Рассматривается задача о рассеянии заряженной квантовой частицы, движущейся в двумерной плоскости трехмерного координатного пространства, на неподвижном кулоновском центре, лежащем в той же плоскости. Выведены и исследованы разложения волновой функции и всех радиальных волновых функций частицы по целым степеням волнового числа и функциям Бесселя вещественного порядка. Доказано, что конечные суммы таких разложений являются асимптотиками в пределе низких энергий.
\end{abstract}

Ключевые слова: двумерное кулоновское рассеяние, волновые функции, низкоэнергетические асимптотики.

DOI: $10.4213 /$ tmf9026

\section{1. ВВЕДЕНИЕ}

В современной теории рассеяния [1] принято использовать термины “двумерный атом водорода" и "двумерное резерфордовское рассеяние". Первый из них означает связанное состояние заряженной квантовой частицы $p_{1}$, взаимодействующей только в двумерной плоскости $\mathcal{P}$ с неподвижным кулоновским силовым центром $O$ посредством потенциала $z_{1} Z / r$, где по определению $z_{1}$ и $Z$ - противоположные по знаку кулоновские заряды частицы и силового центра, a $r$ - расстояние между ними в плоскости $\mathcal{P}$. Под вторым термином подразумевается кулоновское рассеяние частицы $p_{1}$ силовым центром $O$ в той же плоскости $\mathcal{P}$, причем не только в случае кулоновского притяжения $\left(z_{1} Z<0\right)$, но и в случае кулоновского отталкивания $\left(z_{1} Z>0\right)$.

Двумерный атом водорода изучен довольно подробно. Известно точное и исчерпывающее решение задач на связанные состояния для двумерных уравнений Шредингера [2], [3] и Дирака [4], [5] с потенциалом $z_{1} Z / r$. Для всего спектра нерелятивистских связанных состояний в работе [3] вычислены дипольные матричные элементы; выведены формулы, определяющие вероятность одно- и двухфотонных переходов между такими состояниями; исследованы сдвиги собственных значений,

* Объединенный институт ядерных исследований, Дубна, Московская обл., Россия. E-mail: pupyshev@theor.jinr.ru 
порожденные включением постоянного электрического поля, и выявлено сверхтонкое расщепление собственных значений. Анализ спектра двумерного атома водорода в сильном магнитном поле выполнен в работе [6].

Двумерное резерфордовское рассеяние исследовано не столь детально, как двумерный атом водорода. При любой положительной энергии $E$ квантовой частицы известны точные регулярные решения $\Psi^{\mathrm{c}}$ и $\widetilde{\Psi}^{\mathrm{c}}$ задач рассеяния для двумерных уравнений Шредингера [7], [8] и Дирака [9] с потенциалом $z_{1} Z / r$. Аналитические продолжения таких решений в область комплексных значений энергии $E$ неизвестны. Кулоновская функция Грина $G^{\mathrm{c}}$, соответствующая нерелятивистской волновой функции $\Psi^{\mathrm{c}}$ рассеяния квантовой частицы $p_{1}$, исследовалась в работах [10] и [11]. Функцию $G^{\mathrm{c}}$ в импульсном пространстве удалось выразить через элементарные функции [10], а в координатном пространстве записать в виде однократного интеграла [11]. Оценки функции $G^{\text {c }}$ снизу и сверху и ее представление в координатном пространстве через известные специальные функции до сих пор не выведены.

Давно известно [2], что двумерное уравнение Шредингера с потенциалом $z_{1} Z / r$ допускает разделение переменных в полярных координатах $(r, \varphi)$ и с помощью проецирования на базис $\cos m \varphi, m=0,1, \ldots$, сводится к счетной совокупности незацепляющихся одномерных уравнений Шредингера на полупрямой $r>0$. В работе [12] дан анализ регулярного $F_{\lambda}$ и нерегулярного $G_{\lambda}$ решений таких уравнений (здесь $\lambda \equiv m-1 / 2)$.

Низкоэнергетические (при $E \rightarrow+0$ ) асимптотики волновых функций $\Psi^{\mathrm{c}}, F_{\lambda}$ и $G_{\lambda}$ ранее не исследовались. Главная цель настоящей работы - построение и исследование таких асимптотик в области $0 \leqslant r<\infty$. В разделе 2 перечисляются основные определения и обозначения. Низкоэнергетические асимптотики регулярных и нерегулярных волновых функций Кулона $F_{\lambda}$ и $G_{\lambda}$ полуцелого порядка выводятся в разделах 3 и 4. Раздел 5 посвящен построению низкоэнергетической асимптотики волновой функции $\Psi^{\mathrm{c}}$. В заключении перечисляются основные результаты.

\section{2. ОПРЕДЕЛЕНИЯ И ОБОЗНАЧЕНИЯ}

Пусть $\hbar$ - постоянная Планка, $m_{1}$ - масса квантовой частицы $p_{1}$, a $(r, \varphi)-$ ее полярные координаты в плоскости $\mathcal{P}$. Положим

$$
\begin{gathered}
k \equiv \sqrt{\frac{2 m_{1} E}{\hbar^{2}}}, \quad R \equiv \frac{\hbar^{2}}{2 m_{1} z_{1} Z}, \quad \alpha \equiv \operatorname{sgn} R, \quad \eta \equiv \frac{1}{2 k R}=\frac{\alpha}{2 q}, \\
x \equiv \frac{r}{|R|}, \quad \rho \equiv k r=q x, \quad v \equiv \sqrt{8|\eta| \rho}=2 \sqrt{x}, \quad w \equiv v \sin \frac{\varphi}{2}=2 \sqrt{x} \sin \frac{\varphi}{2} .
\end{gathered}
$$

В этих формулах и далее $k$ и $q$ - размерное и безразмерное волновые числа частицы $p_{1}, \eta$ - параметр Зоммерфельда, $x, \rho, v$ и $w$ - безразмерные неотрицательные переменные.

Как пояснялось в работе [11], волновая функция $\Psi^{\mathrm{c}}(\rho, \varphi, \eta)$ двумерного кулоновского рассеяния частицы $p_{1}$ в плоскости $\mathcal{P}$ подчиняется уравнению Шредингера

$$
\left[\partial_{\rho}^{2}+\frac{1}{\rho} \partial_{r}+\frac{1}{\rho^{2}} \partial_{\varphi}^{2}-\frac{2 \eta}{\rho}\right] \Psi^{\mathrm{c}}(\rho, \varphi, \eta)=-\Psi^{\mathrm{c}}(\rho, \varphi, \eta)
$$

с граничным условием

$$
\Psi^{\mathrm{c}}(\rho, \varphi, \eta) \sim e^{i \rho \cos \varphi+i \eta \ln [\rho(1-\cos \varphi)]}+r^{-1 / 2} e^{i \rho-i \eta \ln 2 \rho} f^{\mathrm{c}}(\varphi ; k, \eta), \quad \rho \rightarrow \infty .
$$


Сумма всех операторов, заключенная в уравнении (1) в квадратные скобки, является полным гамильтонианом $H^{\text {c }}$. Он коммутирует с оператором дифференцирования $\partial_{\varphi}^{2}$. Поэтому множество $e^{i m \varphi}, m=0, \pm 1 \ldots$, всех собственных функций оператора $\partial_{\varphi}^{2}$ является полным базисом по угловой переменной. Следовательно, волновую функцию $\Psi^{\mathrm{c}}$ можно представить в виде разложения по такому базису:

$$
\Psi^{\mathrm{c}}(\rho, \varphi, \eta)=\frac{1}{\sqrt{2 \pi \rho}}\left\{\sum_{m=-\infty}^{\infty} u_{m}(\rho, \eta) \cos m \varphi+i \sum_{\substack{n=-\infty \\ n \neq 0}}^{\infty} \tilde{u}_{n}(\rho, \eta) \sin n \varphi\right\} .
$$

Пусть $P_{\varphi}-$ оператор инверсии угла $\varphi$, действующий на любую функцию $B(\rho, \varphi, \eta)$ по правилу $P_{\varphi} B(\rho, \varphi, \eta)=B(\rho,-\varphi, \eta)$. Оператор $P_{\varphi}$ имеет два собственных числа $p_{\varphi}= \pm 1$ и коммутирует с гамильтонианом $H^{\mathrm{c}}$. Поэтому четность $p_{\varphi}$ относительно инверсии $\varphi \rightarrow-\varphi$ является сохраняющимся квантовым числом. Искомая волновая функция $\Psi^{\mathrm{c}}$ имеет четную $\left(p_{\varphi}=+1\right)$ относительно такой инверсии асимптотику $(2)$ и, следовательно, является четной функцией угла $\varphi$ во всей плоскости $\mathcal{P}$. Поэтому любая проекция $\tilde{u}_{n}$ функции $\Psi^{\mathrm{c}}$ на нечетную функцию $\sin n \varphi$ угла $\varphi$ тождественно равна нулю, а разложение (3) вырождается в косинус-ряд Фурье:

$$
\Psi^{\mathrm{c}}(\rho, \varphi, \eta)=\frac{1}{\sqrt{2 \pi \rho}}\left\{u_{0}(\rho, \eta)+2 \sum_{m=1}^{\infty} u_{m}(\rho, \eta) \cos m \varphi\right\} .
$$

Следуя работам [11], [12], вместо целого индекса $m=0,1, \ldots$ введем полуцелый индекс $\lambda=m-1 / 2$ и запишем косинус-ряд Фурье функции $\Psi^{\mathrm{c}}$ в следующем виде:

$$
\Psi^{\mathrm{c}}(\rho, \varphi, \eta)=\frac{1}{\sqrt{2 \pi \rho}}\left\{u_{-1 / 2}(\rho, \eta)+2 \sum_{2 \lambda=1}^{\infty} u_{\lambda}(\rho, \eta) \cos \frac{(2 \lambda+1) \varphi}{2}\right\} .
$$

В уравнении Шредингера (1) заменим искомую функцию $\Psi^{\mathrm{c}}$ ее разложением (4). Полученное уравнение спроецируем на каждую базисную функцию $\cos [(2 \lambda+1) \varphi / 2]$ и таким образом выведем счетную $(2 \lambda=-1,1,3, \ldots)$ последовательность не зацепляющихся друг за друга уравнений Кулона с полуцелым индексом $\lambda$ :

$$
\left[\partial_{\rho}^{2}-\frac{\lambda(\lambda+1)}{\rho^{2}}+1-\frac{2 \eta}{\rho}\right] u_{\lambda}(\rho, \eta)=0, \quad \rho>0, \quad \eta \in(-\infty, \infty) .
$$

Для уравнений Шредингера (1) и Кулона (5) пределом нулевой энергии мы назовем предел $E \rightarrow+0$ при фиксированных значениях параметра $R \neq 0$ и порядка $\lambda \geqslant-1 / 2$. В таком пределе оба волновых числа $k$ и $q$ сходятся к нулю справа, а модуль параметра Зоммерфельда $\eta$ неограниченно возрастает. Под низкоэнергетической асимптотикой исследуемой кулоновской функции мы подразумеваем ее асимптотику в пределе нулевой энергии рассеяния, понимаемом в указанном выше смысле.

Напомним определение ряда Маклорена [13]. Пусть функция $T(q)$ имеет непрерывные производные порядка $p=0,1, \ldots, n$ на отрезке $[0, b]$, где $0<b<\infty$. Тогда ее можно представить в виде ряда Тейлора с центром в точке $q=0$ :

$$
T(q)=\left.\sum_{p=0}^{n-1} \frac{q^{p}}{p !} \partial_{q}^{p} T(q)\right|_{q=0}+R_{n}(q, t), \quad R_{n}(q, t)=\left.\frac{q^{n}}{n !} \partial_{q}^{n} T(q)\right|_{q=t} .
$$


Здесь $q \in[0, b], t \in(0, b)$. Такой ряд называется рядом Маклорена. В пределе $q \rightarrow 0$ его остаточное слагаемое $R_{n}(q, t)$ убывает, как $O\left(q^{n}\right)$. Если $T(-q)=T(q), q \in[0, b]$, то ряд Маклорена содержит только четные степени аргумента $q$.

Будем использовать известный в теории рядов [14] признак равномерной сходимости бесконечного ряда $S$ : если ряд $\widetilde{S}$, все слагаемые которого положительные, превосходит модуль ряда $S$ и сходится, то ряд $S$ сходится равномерно. Ряд $\widetilde{S}$ принято называть мажорантой ряда $S$.

Ключевым в наших рассуждениях является известный асимптотический метод [15] построения решения дифференциального уравнения второго порядка в виде бесконечного разложения по известным специальным функциям.

Следует различать два случая: случай $\alpha=1$ кулоновского отталкивания и случай $\alpha=-1$ кулоновского притяжения. Для краткости далее мы используем символы и формулы с верхними и нижними знаками плюс и (или) минус, подразумевая, что в таких формулах все верхние знаки берутся в случае $\alpha=1$, а все нижние - в случае $\alpha=-1$. Например, формула

$$
A^{ \pm}=a^{ \pm}+\left\{\begin{array}{l}
b \\
c
\end{array}\right\}
$$

означает, что в случае $\alpha=1$ верно равенство $A^{+}=a^{+}+b$, а в случае $\alpha=-1$ верно равенство $A^{-}=a^{-}+c$.

\section{3. НИЗКОЭНЕРГЕТИЧЕСКИЕ АСИМПТОТИКИ РЕГУЛЯРНЫХ ФУНКЦИЙ КУЛОНА}

В настоящем разделе регулярные функции Кулона $F_{\lambda}$ считаются функциями переменных $v$ и q. В п. 3.1 приводятся известные представления [12] этих функций через функции Куммера [16]-[18]. Пункт 3.2 содержит вывод формул дифференцирования функций Куммера по переменной q. В п. 3.3 эти формулы применяются, чтобы представить функции $F_{\lambda}$ в виде рядов Маклорена по переменной $q$. Эти конечные ряды позволят нам далее построить искомые представления функций $F_{\lambda}$ в виде бесконечных рядов, содержащих заранее неизвестные числовые коэффициенты $b_{n s}$, функции Бесселя [16]-[18] первого рода $J_{\mu}(v)$ и $I_{\mu}(v)$ и целые степени переменных $v$ и $q$. В п. 3.4 выводится и исследуется цепочка рекуррентных уравнений для коэффициентов $b_{n s}$. Свойства этих коэффициентов используются в п. 3.5 для доказательства и анализа сходимости полученных разложений функций $F_{\lambda}$ по функциям Бесселя.

3.1. Представления и свойства регулярных функций Кулона. Регулярные волновые функции Кулона $F_{\lambda}$ полуцелого порядка $\lambda$ подробно обсуждались в работе [12]. Напомним определение функций $F_{\lambda}$ и приведем некоторые формулы из этой работы.

Регулярной волновой функцией Кулона полуцелого порядка $\lambda=-1 / 2,1 / 2, \ldots$ называется вещественное решение $F_{\lambda}(\rho, \eta)$ уравнения $(5)$, обладающее асимптотиками

$$
\begin{array}{ll}
F_{\lambda}(\rho, \eta)=O\left(\rho^{\lambda+1}\right), & \rho \rightarrow 0, \\
F_{\lambda}(\rho, \eta)=\sin \left[\rho-\eta \ln 2 \rho-\pi \frac{\lambda}{2}+\delta_{\lambda}^{\mathrm{c}}(\eta)\right]+O\left(\frac{1}{\rho}\right), & \rho \rightarrow \infty
\end{array}
$$


где $\delta_{\lambda}^{\mathrm{c}}(\eta)$ - кулоновская фаза, равная аргументу гамма-функции $\Gamma(1+\lambda+i \eta)$. Функция $F_{\lambda}$ выражается через функцию Куммера [16]

$$
\Phi(\alpha, \gamma ; z)=\sum_{n=0}^{\infty} \frac{(\alpha)_{n}}{(\gamma)_{n}} \frac{z^{n}}{n !}, \quad(\alpha)_{n}=\alpha(\alpha+1) \ldots(\alpha+n-1), \quad(\alpha)_{0}=1
$$

следующим образом:

$$
F_{\lambda}(\rho, \eta)=C_{\lambda}(\eta) \rho^{\lambda+1} e^{i \rho} \Phi(\lambda+1+i \eta, 2 \lambda+2,-2 i \rho) .
$$

Эта формула содержит кулоновский множитель

$$
C_{\lambda}(\eta)=2^{\lambda} \frac{|\Gamma(\lambda+1+i \eta)|}{\Gamma(2 \lambda+2)} e^{-\pi \eta / 2}
$$

и после замены функции $\Phi$ ее разложением в степенной ряд приобретает вид

$$
F_{\lambda}(\rho, \eta)=C_{\lambda}(\eta) \rho^{\lambda+1} e^{i \rho} \sum_{n=0}^{\infty} \frac{(\lambda+1+i \eta)_{n}}{(2 \lambda+2)_{n}} \frac{(-2 i \rho)^{n}}{n !} .
$$

Из этого равенства следует представление

$$
F_{\lambda}(\rho, \pm|\eta|)=q^{\lambda+1} C_{\lambda}(\eta)(2 \lambda+1) ! \frac{v}{2} f_{\lambda}^{ \pm}(v, q)
$$

в котором

$$
f_{\lambda}^{ \pm}(v, q) \equiv e^{i q v^{2} / 4}\left(\frac{v}{2}\right)^{\nu} \sum_{n=0}^{\infty} \frac{(\lambda+1 \pm i / 2 q)_{n}}{\Gamma(\nu+n+1) n !}\left(-2 i q \frac{v^{2}}{4}\right)^{n}, \quad \nu \equiv 2 \lambda+1 .
$$

В отличие от функции $F_{\lambda}$ функция $f_{\lambda}^{ \pm}$является четной функцией волнового числа $q$. Это свойство сыграет важную роль в п. 3.3 и 3.4 .

3.2. Ряды Маклорена для функций Куммера. В соответствии с представлением $(6),(7)$ волновая функция $F_{\lambda}$ является произведением, один из множителей которого - функция Куммера $\Phi(a, c ; \xi)$. Ее параметр $a=\lambda+1+i \eta$, где $\eta=\alpha / 2 q$, и аргумент $\xi=-i 2 \rho=-i 2 q x$ зависят от волнового числа $q$, а параметр $c=2 \lambda+2$ не зависит от этого числа. Ниже нам потребуются производные $\partial_{q}^{n} \Phi(a, c ; \xi), n=0,1, \ldots$, функции $\Phi(a, c ; \xi)$ по волновому числу $q$ в точке $q=0$. Такие производные нетрудно вычислить, если найдены производные этой же функции $\Phi(a, c ; \xi)$ по параметру $a$ и по аргументу $\xi$. В теории специальных функций [16] известны формулы дифференцирования функции Куммера по аргументу, но формулы дифференцирования по параметрам неизвестны. Поэтому для вычисления производных $\partial_{q}^{n} \Phi(a, c ; \xi)$, $n=0,1, \ldots$, мы предлагаем довольно простой способ. Опишем его.

Представим исследуемую функцию Куммера в виде суммы:

$$
\Phi(\lambda+1+i \alpha|\eta|, 2 \lambda+2,-2 i \rho)=\nu ! \sum_{n=0}^{\infty} \frac{(\lambda+1+i \alpha / 2 q)_{n}}{\Gamma(\nu+n+1) n !}\left(-2 i q \frac{v^{2}}{4}\right)^{n} .
$$

Каждое ее слагаемое содержит произведение двух множителей, зависящих от переменной $q$. Запишем такое произведение в виде

$$
\left(\lambda+1+\frac{i \alpha}{2 q}\right)_{n}\left(-2 i q \frac{v^{2}}{4}\right)^{n}=\left(\alpha \frac{v^{2}}{4}\right)^{n} A_{n}(t ; \lambda), \quad t \equiv \frac{2 q}{i \alpha}=-2 i \alpha q .
$$


Теперь решим следующую задачу: найти коэффициенты $a_{p}, p \leqslant 4$, полинома

$$
A_{n}(t ; \lambda) \equiv \prod_{j=1}^{n}[(\lambda+j) t+1]=1+a_{1} q+a_{2} q^{2}+\cdots+a_{n} q^{n} .
$$

Все коэффициенты $a_{p}$ являются функциями $a_{p}(\lambda, n, \alpha)$ трех дискретных аргументов $\lambda, n=1,2, \ldots$ и $\alpha= \pm 1$. Для сокращения записи эти аргументы мы далее не указываем. Коэффициенты $a_{p}$ связаны с производными полинома $A_{n}$ по переменной $t$ :

$$
a_{p}=\left.\frac{(-2 i \alpha)^{p}}{p !} \partial_{t}^{p} A_{n}(t ; \lambda)\right|_{t=0}, \quad p=1,2, \ldots .
$$

Для вычисления таких производных введем суммы

$$
S_{m}(t ; \lambda) \equiv \sum_{j=1}^{n} \frac{(\lambda+j)^{m}}{[(\lambda+j) t+1]^{m}}, \quad m=1,2,3,4 .
$$

Эти суммы обладают двумя свойствами: при любых $t$ и $\lambda$ верны соотношения

$$
\partial_{t} S_{1}(t ; \lambda)=-S_{2}(t ; \lambda), \quad \partial_{t} S_{2}(t ; \lambda)=-2 S_{3}(t ; \lambda), \quad \partial_{t} S_{3}(t ; \lambda)=-3 S_{4}(t ; \lambda),
$$

а в частном случае $t=0$ имеют место представления

$$
\begin{aligned}
& S_{1}(t ; \lambda)=n \lambda+s_{1}, \\
& S_{2}(t ; \lambda)=n \lambda^{2}+2 \lambda s_{1}+s_{2}, \\
& S_{3}(t ; \lambda)=n \lambda^{3}+3 \lambda\left(\lambda s_{1}+s_{2}\right)+s_{3}, \\
& S_{4}(t ; \lambda)=n \lambda^{4}+4 \lambda\left(\lambda^{2} s_{1}+s_{3}\right)+6 \lambda^{2} s_{2}+s_{4}
\end{aligned}
$$

через известные табличные суммы [19]

$$
\begin{gathered}
s_{m} \equiv \sum_{j=1}^{n} j^{m}, \quad m=1,2,3,4 ; \\
s_{1}=\frac{1}{2} n(n+1), \quad s_{2}=\frac{1}{6} n(n+1)(2 n+1), \\
s_{3}=\frac{1}{2} n^{2}(n+1)^{2}, \quad s_{4}=\frac{n}{30}(n+1)(2 n+1)\left(3 n^{2}+3 n-1\right) .
\end{gathered}
$$

Используя первое из указанных выше свойств сумм $S_{m}$ и последовательно увеличивая порядок $p=1,2,3,4$ производных $\partial_{t}^{p} A_{n}$, выведем следующие равенства:

$$
\begin{aligned}
& \partial_{t} A_{n}=A_{n} S_{1}, \quad \partial_{t}^{2} A_{n}=A_{n}\left(S_{1}^{2}-S_{2}\right), \quad \partial_{t}^{3} A_{n}=A_{n}\left(S_{1}^{3}-3 S_{1} S_{2}+2 S_{3}\right), \\
& \partial_{t}^{4} A_{n}=A_{n}\left(S_{1}^{4}-6 S_{1}^{2} S_{2}+3 S_{2}^{2}+8 S_{1} S_{3}-6 S_{4}\right)
\end{aligned}
$$

где для краткости записи аргумент $t$ и параметр $\lambda$ опущены. Теперь в этих равенствах положим $t=0$ и применим второе свойство сумм $S_{m}$. В результате найдем производные $\partial_{t}^{p} A_{n}(t ; \lambda), p=1,2,3,4$, в точке $t=0$. Затем по формулам (14) получим искомые представления для коэффициентов $a_{p}$ полинома $A_{n}(t ; \lambda)$. Положив 
$a \equiv 2 \lambda+n+1$, запишем эти представления:

$$
\begin{gathered}
a_{1}=-i \alpha a n, \quad a_{2}=-\frac{n}{6}(n-1)\left(n+1-3 a^{2}\right), \\
a_{3}=i \alpha a \frac{n}{6}(n-1)(n-2)\left(n+1-a^{2}\right), \\
a_{4}=\frac{n}{360}(n-1)(n-2)(n-3)\left[15 a^{2}\left(a^{2}-2 a-4 \lambda\right)+(n+1)(5 n+7)\right] .
\end{gathered}
$$

Итак, доказано вспомогательное разложение

$$
\left(\lambda+1+\frac{i \alpha}{2 q}\right)_{n}\left(-2 i q \frac{v^{2}}{4}\right)^{n}=\left(\alpha \frac{v^{2}}{4}\right)^{n}\left[1+\sum_{p=1}^{m} a_{p} q^{p}+O\left(q^{m+1}\right)\right],
$$

где $m=1,2,3,4$, а $a_{p}$ - функции (18) дискретных переменных $\lambda, n$ и $\alpha$. Используя это разложение, нетрудно выразить производные $\partial_{q}^{m} \Phi$ исследуемой функции Куммера (11) в точке $q=0$ для $m \leqslant 4$ через функции Бесселя первого рода $J_{\mu}$ и $I_{\mu}$.

Для примера найдем производную первого порядка в точке $q=0$. В ряде (11) заменим все произведения множителей, зависящих от переменной $q$, правыми частями равенств (19). Полученный ряд продифференцируем почленно по аргументу $q$. Положим $q=0$. Коэффициент $a_{1}$ выразим по первой из формул (18) через дискретные аргументы $\lambda, n$ и $\alpha$. Итоговое соотношение запишем в виде равенства

$$
\left.\partial_{q} \Phi\right|_{q=0}=-i \alpha \nu !\left\{\sum_{n=0}^{\infty} \frac{(2 \lambda+2) n+n(n-1)}{\Gamma(\nu+1+n) n !}\left(\alpha \frac{v^{2}}{4}\right)^{n}\right\}, \quad \nu=2 \lambda+1 .
$$

Это равенство с помощью двух $(m=0,1)$ однотипных цепочек преобразований

$$
\begin{aligned}
& \sum_{n=0}^{\infty} \frac{\left(\alpha v^{2} / 4\right)^{n}}{\Gamma(\nu+n+1)} \frac{n(n-1) \ldots(n-m)}{n !}= \\
& \quad=\sum_{n=m+1}^{\infty} \frac{\left(\alpha v^{2} / 4\right)^{n}}{\Gamma(\nu+n+1)} \frac{1}{(n-m-1) !}=\left(\alpha \frac{v^{2}}{4}\right)^{m+1} \sum_{n=0}^{\infty} \frac{\left(\alpha v^{2} / 4\right)^{n}}{\Gamma(\nu+m+n+1) n !}
\end{aligned}
$$

сведем к сумме двух бесконечных рядов:

$$
\begin{aligned}
& \left.\partial_{q} \Phi\right|_{q=0}= \\
& \quad=-i \alpha \nu !\left(\alpha \frac{v^{2}}{4}\right)\left\{(\nu+1) \sum_{n=0}^{\infty} \frac{\left(\alpha v^{2} / 4\right)^{n}}{\Gamma(\nu+2+n) n !}+\left(\alpha \frac{v^{2}}{4}\right) \sum_{n=0}^{\infty} \frac{\left(\alpha v^{2} / 4\right)^{n}}{\Gamma(\nu+3+n) n !}\right\} .
\end{aligned}
$$

Сравнив эти ряды с известными разложениями [16] функций Бесселя первого рода

$$
\left(\frac{v}{2}\right)^{\mu} \sum_{n=0}^{\infty} \frac{\left( \pm v^{2} / 4\right)^{n}}{\Gamma(n+\mu+1) n !}=Z_{\mu}^{ \pm}(v)=\left\{\begin{array}{l}
I_{\mu}(v) \\
J_{\mu}(v)
\end{array}\right.
$$

заключаем, что имеет место равенство

$$
\left.\partial_{q} \Phi(\lambda+1 \pm|\eta|, 2 \lambda+2,-2 i \rho)\right|_{q=0}=\mp i \nu !\left(\frac{v}{2}\right)^{1-\nu}\left[(\nu+1) Z_{\nu+1}^{ \pm}(v) \pm \frac{v}{2} Z_{\nu+2}^{ \pm}(v)\right] .
$$


Функции Бесселя $Z_{\nu+1}^{ \pm}(v)$ ограничены при $v<\infty$. Следовательно, при $v<\infty$ модуль правой части этого равенства является мажорантой первой производной исходного ряда (11) в точке $q=0$. Поэтому такой ряд сходится равномерно, если $v<\infty$. Таким образом, его почленное дифференцирование, использованное выше, правомерно.

Продолжим исследование производных ряда Куммера (11) по переменной q. Последовательно выведем заведомо завышенные, но зато простые мажорантные оценки сумм $S_{m}(t ; \lambda)$, полинома $A_{n}(t ; \lambda)$ и его производных $\partial_{t}^{m} A_{n}(t ; \lambda)$.

Для начала заметим, что $|(\lambda+j) t+1|>1$ при любых $\lambda, j$ и $t$, поэтому модуль суммы $S_{m}(t ; \lambda)$ никогда не превосходит значения этой же суммы в точке $t=0$ :

$$
\left|S_{m}(t ; \lambda)\right| \leqslant\left. S_{m}(t ; \lambda)\right|_{t=0}, \quad m=1,2,3,4 .
$$

Далее, из определения (13) полинома $A_{n}$ и соотношений

$$
|(\lambda+j) t+1|=|(\lambda+j)(-2 i \alpha q)+1|=\left[4(\lambda+j)^{2} q^{2}+1\right]^{1 / 2} \leqslant 2(\lambda+j) q+1
$$

следует, что для $\lambda \geqslant-1, n=0,1, \ldots$ и $q \geqslant 0$ верно неравенство

$$
\left|\left(\alpha \frac{v^{2}}{4}\right)^{n} A_{n}(t ; \lambda)\right| \leqslant(\lambda+1+|\eta|)_{n}(2 \rho)^{n} .
$$

Используя упомянутые выше неравенства, из представлений (17) производных полинома $A_{n}$ получаем следующие справедливые при любых $t, n$ и $\lambda$ оценки:

$$
\begin{aligned}
& \left|\partial_{q} A_{n}(t ; \lambda)\right| \leqslant(\lambda+1+|\eta|)_{n}(2 \rho)^{n} S_{1}, \\
& \left|\partial_{q}^{2} A_{n}(t ; \lambda)\right| \leqslant 2(\lambda+1+|\eta|)_{n}(2 \rho)^{n}\left(S_{1}^{2}+S_{2}\right), \\
& \left|\partial_{q}^{3} A_{n}(t ; \lambda)\right| \leqslant 8(\lambda+1+|\eta|)_{n}(2 \rho)^{n}\left(S_{1}^{3}+3 S_{1} S_{2}+2 S_{3}\right), \\
& \left|\partial_{q}^{4} A_{n}(t ; \lambda)\right| \leqslant 16(\lambda+1+|\eta|)_{n}(2 \rho)^{n}\left(S_{1}^{4}+6 S_{1}^{2} S_{2}+3 S_{2}^{2}+8 S_{1} S_{3}+6 S_{4}\right) .
\end{aligned}
$$

В этих оценках символами $S_{m}$ обозначены суммы $S_{m}(t ; \lambda)$, взятые в точке $t=0$. Такие суммы нетрудно выразить через индексы $\lambda, n$ и $m$ по формулам (15) и (16).

Теперь решим следующую задачу: используя первую из оценок (22), показать, что функция Куммера $\Phi$, заданная равенством (11), имеет непрерывную производную $\partial_{q} \Phi$ при любых конечных значениях аргументов $q$ и $v$.

Для решения этой задачи сначала в исходном ряде Куммера (11) заменим все произведения множителей, зависящих от переменной $q$, правыми частями равенств (12). Полученный ряд продифференцируем почленно по аргументу $q$ и таким образом получим ряд $\partial_{q} \Phi$, слагаемые которого содержат функции $\partial_{q} A_{n}(q ; \lambda)$. Модуль ряда $\partial_{q} \Phi$ не превосходит ряда Куммера, содержащего вместо функций $\partial_{q} A_{n}$ их модули $\left|\partial_{q} A_{n}\right|$. Каждый такой модуль заменим правой частью первого из неравенств $(22)$, в котором предварительно выразим сумму $S_{1}$ через индексы $\lambda$ и $n$ по формулам (15) и (16). Полученный ряд обозначим как $Q$ и представим равенствами

$$
Q(v, q) \equiv \frac{\nu !}{2} \sum_{n=0}^{\infty} \frac{n(\nu+n)(\lambda+1+|\eta|)_{n}}{\Gamma(\nu+n+1) n !}(2 \rho)^{n}=(\lambda+1+|\eta|) \rho \Phi(\lambda+1+|\eta|, 2 \lambda+2 ; 2 \rho) .
$$


Заметим, что в этих равенствах согласно справочнику [16] функция Куммера неограничена только в пределе $\rho \rightarrow \infty$. Поэтому аналогичным свойством обладает и функция $Q$. По построению эта функция является верхней оценкой ряда $\left|\partial_{q} \Phi\right|$. Следовательно, если $\rho<\infty$, то исходный ряд $\partial_{q} \Phi$ сходится равномерно и является непрерывной функцией переменной $q$, что и требовалось показать.

Сделаем несколько полезных замечаний.

С помощью оценок (22) производных полинома $A_{n}$ несложно доказать, что производные $\partial_{q}^{m} \Phi, m=2,3,4$, исследуемой функции Куммера (11) непрерывны и ограничены при любых конечных значениях аргументов $v$ и $q$. Доказательство принципиально не отличается от рассмотренного выше случая $m=1$.

Предложенный и поясненный в настоящем пункте метод можно обобщить на случай $m>4$ и убедиться в том, что функция Куммера (11) имеет непрерывные по переменной $q$ производные любого конечного порядка $m$ при $q<\infty, v<\infty$. Поэтому при $q<\infty, v<\infty$ и любом $m<\infty$ для такой функций существует ряд Маклорена, содержащий производные $\partial_{q}^{p} \Phi, p \leqslant m$, в точке $q=0$ и остаточное слагаемое, которое в пределе $q \rightarrow+0$ убывает, как $O\left(q^{m+1}\right)$. Упомянутое обобщение - довольно трудоемкое занятие. Более простой способ разложения функции Куммера (11) в ряд Маклорена с большим числом слагаемых $(m>4)$ основан на методе, предложенном ниже в п. 5.2 .

3.3. Ряды Маклорена для регулярных функций Кулона. Как отмечалось в п. 3.1, $f_{\lambda}^{ \pm}(v, q)$ - четная функция (10) переменной $q$. Следовательно, ряд Маклорена функции $f_{\lambda}^{ \pm}(v, q)$ по переменной $q$ при любом числе $m=0,1, \ldots$ слагаемых должен содержать только слагаемые с четными степенями $2 m$ переменной $q$. Проверим это следствие в трех случаях $m=0, m=1$ и $m=2$. Для этого построим ряды Маклорена функции $f_{\lambda}^{ \pm}(v, q)$ по ее аргументу $q$. Используем все результаты предыдущего пункта.

Приступим к построению. Сначала в разложении (10) функции $f_{\lambda}^{ \pm}$заменим экспоненциальный множитель его известным рядом Маклорена [13],

$$
e^{i \rho}=e^{i q v^{2} / 4}=1+\sum_{p=1}^{4} \frac{(i q)^{p}}{p !}\left(\frac{v^{2}}{4}\right)^{p}+O\left(q^{5}\right), \quad v<\infty
$$

а каждое слагаемое, стоящее под знаком суммы, преобразуем по формуле (12), в которой заменим полином $A_{n}$ суммой (13) с коэффициентами $a_{n}$, заданными формулами (18). Затем в полученном представлении функции $f_{\lambda}^{ \pm}$приведем подобные члены с одинаковой зависимостью от переменной $q$ и каждый из них соответствующей цепочкой преобразований (20) сведем к произведениям целой степени переменной $v$ и функции Бесселя $I_{\mu}(v)$ или $J_{\mu}(v)$. В результате получим три $(m=0,1,2)$ разложения функции $f_{\lambda}^{ \pm}$:

$$
f_{\lambda}^{ \pm}(v, q)=f_{\lambda m}^{ \pm}(v, q)+R_{\lambda m}^{ \pm}(v, q)
$$

В каждом из них $R_{\lambda m}^{ \pm}=O\left(q^{m+1}\right)$ - остаточный член, а $f_{\lambda m}^{ \pm}$- конечная сумма,

$$
f_{\lambda m}^{ \pm}(v, q)=\sum_{n=0}^{m}\left(-\frac{q^{2}}{8}\right)^{n} \frac{1}{n !} \sum_{s=2 n}^{3 n} b_{n s} v^{s}\left\{\begin{array}{c}
(-1)^{s} I_{\nu+s}(v) \\
J_{\nu+s}(v)
\end{array}\right\}
$$


в которой коэффициенты $b_{n s}$ вычисляются по формулам

$$
\begin{gathered}
b_{00}=1, \quad b_{12}=\nu+1, \quad b_{13}=\frac{1}{3}, \\
b_{24}=(\nu+3)(\nu+1), \quad b_{25}=\frac{2}{3}\left(\nu+\frac{11}{5}\right), \quad b_{26}=\frac{1}{9}
\end{gathered}
$$

и поэтому зависят только от целых индексов $n$ и $s$ и целого числа $\nu=2 \lambda+1 \geqslant 0$.

Теперь в формуле (8) заменим функцию $f_{\lambda}^{ \pm}$суммой (24) со слагаемыми (25) и таким образом для функций $F_{\lambda}$ выведем искомые ряды Маклорена:

$$
F_{\lambda}(\rho, \pm|\eta|)=F_{\lambda m}(\rho, \pm|\eta|)+q^{\lambda+1} C_{\lambda}(\eta) \nu ! \frac{v}{2} R_{\lambda m}^{ \pm}(v, q),
$$

где $m=0,1,2$ и для краткости записи мы положили

$$
\begin{aligned}
F_{\lambda m}(\rho, \pm|\eta|) & \equiv q^{\lambda+1} C_{\lambda}(\eta) \nu ! \frac{v}{2} f_{\lambda m}^{ \pm}(v, q)= \\
& =q^{\lambda+1} C_{\lambda}(\eta) \nu ! \frac{v}{2} \sum_{n=0}^{m}\left(-\frac{q^{2}}{8}\right)^{n} \frac{1}{n !} \sum_{s=2 n}^{3 n} b_{n s} v^{s}\left\{\begin{array}{c}
(-1)^{s} I_{\nu+s}(v) \\
J_{\nu+s}(v)
\end{array}\right\} .
\end{aligned}
$$

Согласно выводам, сделанным в конце п. 3.2, разложения $(27),(28)$ функций $F_{\lambda}$ являются рядами Маклорена при $q<\infty$ и $v<\infty$, а в пределе $q \rightarrow+0$ функция $R_{\lambda m}$ сходятся к нулю, как $O\left(q^{m+1}\right)$, если $v<\infty$. Поэтому функции $F_{\lambda m}, m=0,1,2$, являются низкоэнергетическими асимптотиками функции $F_{\lambda}(\rho, \eta)$ в области $\rho<\infty$.

Изложенный выше способ разложения функций Кулона $F_{\lambda}$ в ряды Маклорена непрактичен при большом числе слагаемых таких рядов. В этом случае выгоднее использовать метод, предложенный в следующем пункте.

\section{4. Разложения регулярных функций Кулона по функциям Бесселя.} Построение разложений регулярных функций Кулона $F_{\lambda}$ по функциям Бесселя $I_{\mu}$ или $J_{\mu}$ начнем с вывода и качественного анализа вспомогательного уравнения.

Подстановками

$$
u_{\lambda}(\rho, \pm|\eta|)=v y_{\nu}^{ \pm}(v, \tau), \quad \tau \equiv\left(\frac{q}{2}\right)^{2}, \quad \nu=2 \lambda+1, \quad v=\sqrt{2|\eta| \rho}=2 \sqrt{x}
$$

сведем исходное уравнение Кулона (5) к уравнению

$$
\left[\partial_{v}^{2}+\frac{1}{v} \partial_{v} \mp 1-\frac{\nu^{2}}{v^{2}}+\tau v^{2}\right] y_{\nu}^{ \pm}(v, \tau)=0, \quad v>0 .
$$

При $\tau=0$ данное уравнение вырождается в уравнение Бесселя целого порядка $\nu=2 \lambda+1$. Если $\tau \neq 0$, то формальным решением обсуждаемого уравнения будет бесконечный ряд по целым степеням $n=0,1,2, \ldots$ параметра $\tau$. Чтобы угадать структуру такого разложения искомого регулярного решения $y_{\nu}^{ \pm}=f_{\lambda}^{ \pm}$, вернемся к представлению $(24)-(26)$ функции $f_{\lambda}^{ \pm}$. Это представление позволяет предположить, что функция $f_{\lambda}^{ \pm}$является следующей двойной суммой: в первой бесконечной сумме индекс $n$ принимает значения $0,1, \ldots$, а во второй (конечной) сумме при каждом $n$ индекс суммирования $s$ пробегает все целые значения от $2 n$ до $3 n$ включительно. Воспользуемся этим предположением и следующим замечанием: для искомого регулярного решения $f_{\lambda}^{ \pm}(v, q)$ уравнения (30) сумма трех первых слагаемых 
(при $n=0,1,2)$ бесконечного разложения по целым степеням параметра $\tau$ должна совпадать с функцией $f_{\lambda m}^{ \pm}(v, q)$, заданной формулой $(25)$ при $m=2$. Поэтому решение $f_{\lambda}^{ \pm}$будем искать в виде бесконечного ряда

$$
f_{\lambda}^{ \pm}(v, q)=\sum_{n=0}^{\infty}\left(-\frac{q^{2}}{8}\right)^{n} \frac{1}{n !} \sum_{s=2 n}^{3 n} b_{n s} v^{s}\left\{\begin{array}{c}
(-1)^{s} I_{\nu+s}(v) \\
J_{\nu+s}(v)
\end{array}\right\}
$$

в котором коэффициенты $b_{n s}$ при $n \leqslant 2$ определены формулами $(26)$, а все остальные коэффициенты $b_{n s}$ пока неизвестны.

Докажем, что исследуемый ряд (31) формально удовлетворяет уравнению (30), если коэффициенты $b_{n s}$ подчиняются рекуррентной цепочке уравнений

$$
s b_{n s}=n\left[b_{n-1, s-3}+2(\nu+s-1) b_{n-2, s-2}\right], \quad n=0,1, \ldots, \quad s=2 n, 2 n+1, \ldots, 3 n,
$$

в которой $b_{00}=1$ и $b_{m t}=0$, если $t<2 m$ или $t>3 m$.

Схема доказательства простая, но ее реализация - довольно трудоемкая и малоинтересная процедура. Поэтому ограничимся лишь описанием основных этапов. В уравнении (30) заменим функцию $y_{\nu}^{ \pm}$соответствующим рядом (31). Полученное уравнение запишем в виде полинома по целым степеням переменной $\tau$. Каждый из коэффициентов этого полинома приравняем нулю. Затем, применив известные рекуррентные соотношения для функций Бесселя и их производных [16], убедимся в том, что каждое из полученных равенств, вырождается в тождество по переменной $v$, если коэффициенты $b_{n s}$ подчиняются цепочке уравнений $(32)$. Следовательно, при данном условии ряд (31) представляет собой формальное решение уравнения (30).

Исследуем коэффициенты $b_{n s}$. Считаем, что по определению $(-1) ! !=1$. Заметим, что все коэффициенты (26) удовлетворяют уравнениям (32). Положим в этих уравнениях $s=3 n$, а $n=m, m-1, m-2, \ldots, 1$. В результате получим цепочку равенств

$$
b_{n, 3 n}=\frac{1}{3} b_{n-1,3(n-1)}=\frac{1}{3} \cdot \frac{1}{3} b_{n-2,3(n-2)}=\cdots=\frac{1}{3^{n-1}} b_{13},
$$

последнее из которых содержит коэффициент $b_{13}=1 / 3$ согласно формулам (26). Следовательно, $b_{n, 3 n}=3^{-n}$ при любом $n=1,2, \ldots$. Аналогичным способом, положив $s=2 n, n=m, m-1, \ldots, 1$, из соотношений (26) и (32) получаем

$$
b_{n, 2 n}=\prod_{m=0}^{n-1}\left[(\nu-1+2(n-m)]=\frac{(\nu+2 n-1) ! !}{(\nu-1) ! !}, \quad n=1,2, \ldots\right.
$$

Докажем по индукции два утверждения: все коэффициенты $b_{n s}$ положительные и при каждом $n$ убывают с ростом $s$. В качестве первого этапа индукции заметим, что согласно формулам (26) эти утверждения верны при $n \leqslant 2$.

Докажем первое утверждение. Предположим, что $b_{n-1, s}>0$ при некотором $n$ и любом $s$, таком что $2(n-1) \leqslant s \leqslant 3(n-1)$. Тогда из цепочки уравнений $(32)$ следует, что $b_{n s}>0$ при любом целом $s$, удовлетворяющем условию $2 n \leqslant s \leqslant 3 n$.

Теперь докажем второе утверждение. Предположим, что $b_{n-1, s}>b_{n-1, s^{\prime}}$ при некотором $n$ и при $s<s^{\prime}$. Сравним равенство (32) с равенством, полученным из 
него заменой $s \mapsto s+1$. Для этого последнее запишем как

$$
b_{n, s+1}=\frac{n}{s+1} b_{n-1, s-2}+2 n\left(1+\frac{\nu-1}{s+1}\right) b_{n-1, s-1} .
$$

Согласно нашему предположению $b_{n-1, s-3}>b_{n-1, s-2}$ и $b_{n-1, s-2}>b_{n-1, s-1}$. Следовательно, $b_{n, s+1}<b_{n s}$ при любом $s$, что и требовалось показать.

Суммируя доказанные свойства всех коэффициентов $b_{n s}$, запишем цепочку неравенств

$$
\frac{1}{3^{n}}=b_{n, 3 n}<b_{n, 3 n-1}<\cdots<b_{n, 2 n+1}<b_{n, 2 n}=\frac{(\nu+2 n-1) ! !}{(\nu-1) ! !} .
$$

Следовательно, при данном $n$ из всех коэффициентов $b_{n s}, s=2 n, 2 n+1, \ldots, 3 n$, наибольшим является коэффициент $b_{n, 2 n}$. Это свойство окажется далее ключевым.

\section{5. Свойства разложений регулярных функций Кулона по функциям}

Бесселя. Самое важное свойство разложений $(9)$, (31) функций Кулона $F_{\lambda}$ по функциям Бесселя заключается в их равномерной сходимости. Докажем и исследуем это свойство сначала в случае $\alpha=-1$, а затем в случае $\alpha=1$.

Найдем мажоранту ряда (31) в случае $\alpha=-1$. Для этого рассмотрим ряд, все слагаемые которого равны модулям слагаемых исходного ряда (31). В этом ряде при каждом $n$ заменим все коэффициенты $b_{n s}$ наибольшим из них коэффициентом $b_{n, 2 n}$. Чтобы оценить сверху каждое слагаемое получившегося ряда, последовательно применим неравенство Ватсона [16]

$$
\left|J_{\mu}(v)\right| \leqslant \frac{(v / 2)^{\mu}}{\Gamma(\mu+1)}, \quad \mu \geqslant-\frac{1}{2},
$$

и два очевидных соотношения

$$
\begin{gathered}
\frac{1}{(\nu+2 n+m) !} \leqslant \frac{1}{(\nu+2 n) !} \frac{1}{(\nu+2 n+1)^{m}} \\
\frac{(\nu+2 n-1) ! !}{(\nu-1) ! !} \frac{1}{(\nu+2 n) !}=\frac{1}{\nu !} \prod_{m=0}^{n-1} \frac{1}{\nu+2 n-2 m} \leqslant \frac{1}{\nu !} \frac{1}{(\nu+2)^{n}} .
\end{gathered}
$$

В итоге получим следующую оценку:

$$
\left|f_{\lambda}^{-}(v, q)\right| \leqslant \frac{1}{\nu !}\left(\frac{v}{2}\right)^{\nu} \sum_{n=0}^{\infty}\left(\frac{\rho^{2}}{2(\nu+2)}\right)^{n} \frac{1}{n !} B_{n}(v) .
$$

Заменив в ней конечную сумму $B_{n}(v)$ табличной суммой [19] по формулам

$$
B_{n}(v) \equiv \sum_{m=0}^{n}\left[\frac{v^{2}}{2(\nu+2 n+1)}\right]^{m} \leqslant \sum_{m=0}^{n} t^{m}=\frac{1-t^{n}}{1-t}, \quad t \equiv \frac{v^{2}}{2(\nu+1)}
$$

получим искомую мажоранту бесконечного ряда (31) в случае $\alpha=-1$.

Мажоранту этого ряда в случае $\alpha=1$ построим аналогичным способом, но вместо неравенства Ватсона (34) используем оценки [18]

$$
0 \leqslant I_{\mu}(v) \leqslant \frac{(v / 2)^{\mu}}{\Gamma(\mu+1)} \operatorname{ch} v, \quad \mu>-\frac{1}{2}, \quad v \geqslant 0
$$


Итоговые соотношения, полученные в обоих случаях, представим в виде

$$
\left|f_{\lambda}^{ \pm}(v, q)\right| \leqslant \frac{1}{\nu !}\left(\frac{v}{2}\right)^{\nu} \frac{e^{z}-t e^{z t}}{1-t}\left\{\begin{array}{c}
\operatorname{ch} v \\
1
\end{array}\right\}, \quad z \equiv \frac{\rho^{2}}{2(\nu+2)} .
$$

В силу этой формулы бесконечные ряды $f_{\lambda}^{ \pm}(v, q)$ сходятся равномерно по обоим неотрицательным аргументам $v$ и $q$, если эти аргументы ограничены и $\nu=2 \lambda+1>0$.

Выясним скорость такой сходимости. Для этого оценим разность между бесконечным рядом $f_{\lambda}^{ \pm}(v, q)$ и его частичной суммой $f_{\lambda m}^{ \pm}(v, q)$ порядка $m<\infty$, заданной формулой (25). Способом, использованным при доказательстве неравенств (36), получаем оценку остаточного слагаемого $R_{\lambda m}^{ \pm}$суммы $(24)$ :

$$
\begin{aligned}
\left|R_{\lambda m}^{ \pm}(v, q)\right| & =\left|f_{\lambda}^{ \pm}(v, q)-f_{\lambda m}^{ \pm}(v, q)\right| \leqslant \\
\leqslant \chi(v, q) & \equiv \frac{1}{\nu ! m !}\left(\frac{v}{2}\right)^{\nu} \frac{z^{m}}{1-t_{m}}\left[e^{z}-t_{m}^{m+1} e^{z t_{m}}\right]\left\{\begin{array}{c}
\operatorname{ch} v \\
1
\end{array}\right\}, \quad t_{m} \equiv \frac{v^{2}}{2(\nu+1+2 m)} .
\end{aligned}
$$

Из нее следует, что в пределе $\rho \rightarrow 0$ конечная сумма $f_{\lambda m}^{ \pm}(v, q)$ также приближает ряд $f_{\lambda}^{ \pm}(v, q)$ с точностью порядка $O\left(z^{m}\right)=O\left(\rho^{2 m}\right)$.

Вернемся к представлению (9) функции $F_{\lambda}(\rho, \pm|\eta|)$ через функцию $f_{\lambda}^{ \pm}(v, q)$. Заменив в нем функцию $f_{\lambda}^{ \pm}$бесконечным рядом $(31)$, получим искомое разложение функции Кулона $F_{\lambda}$ по функциям Бесселя:

$$
F_{\lambda}(\rho, \pm|\eta|)=q^{\lambda+1} C_{\lambda}(\eta) \nu ! \frac{v}{2} \sum_{n=0}^{\infty}\left(-\frac{q^{2}}{8}\right)^{n} \frac{1}{n !} \sum_{s=2 n}^{3 n} b_{n s} v^{s}\left\{\begin{array}{c}
(-1)^{s} I_{\nu+s}(v) \\
J_{\nu+s}(v)
\end{array}\right\}
$$

Оценим модуль разности этого ряда и его частичной суммы $F_{\lambda m}, m<\infty$, определенной формулой (28). Применив равенства (27) и (37), находим

$$
\left|F_{\lambda}(\rho, \pm|\eta|)-F_{\lambda m}(\rho, \pm|\eta|)\right| \leqslant q^{\lambda+1} C_{\lambda}(\eta) \nu ! \frac{v}{2} \chi(v, q) .
$$

Следовательно, в пределе $q \rightarrow+0$ функция $F_{\lambda m}$ приближает функцию $F_{\lambda}$ в области $\rho<\infty$ с абсолютной точностью $O\left(q^{\lambda+1} C_{\lambda}(\eta) \rho^{2 m}\right)$.

Для полноты опишем способ численного анализа качества приближения

$$
F_{\lambda}(\rho, \pm|\eta|) \approx F_{\lambda m}(\rho, \pm|\eta|) .
$$

Начнем с обсуждения функции

$$
D_{\lambda m}(\rho, \pm|\eta|, \varepsilon) \equiv \lg \frac{\left|F_{\lambda}(\rho, \pm|\eta|)-F_{\lambda m}(\rho, \pm|\eta|)\right|+\varepsilon}{\left|F_{\lambda}(\rho, \pm|\eta|)\right|+\varepsilon}, \quad \varepsilon>0 .
$$

Функция $D_{\lambda m}$ непрерывна по любому своему аргументу, равна нулю в точке $\rho=0$ и имеет локальный максимум или локальный минимум в той точке, в которой функция $F_{\lambda}$ или разность функций $F_{\lambda}$ и $F_{\lambda m}$ обращается в нуль. В пределе $\varepsilon \rightarrow+0$ обсуждаемая функция $D_{\lambda m}$ есть логарифм относительной точности аппроксимации (40). Следовательно, при достаточно малом, но положительном значении $\varepsilon$ функция $D_{\lambda m}$ близка к такому логарифму.

Наглядное представление об абсолютной и относительной точности аппроксимации (40) в случаях $2 \lambda=-1, \alpha= \pm 1$ и $q=0.1,0.5$ дают рис. 1 и рис. 2 . На рис. 1 изображены графики функции $F_{\lambda}$ и аппроксимирующих ее функций $F_{\lambda m}, m=0,2,5$, 

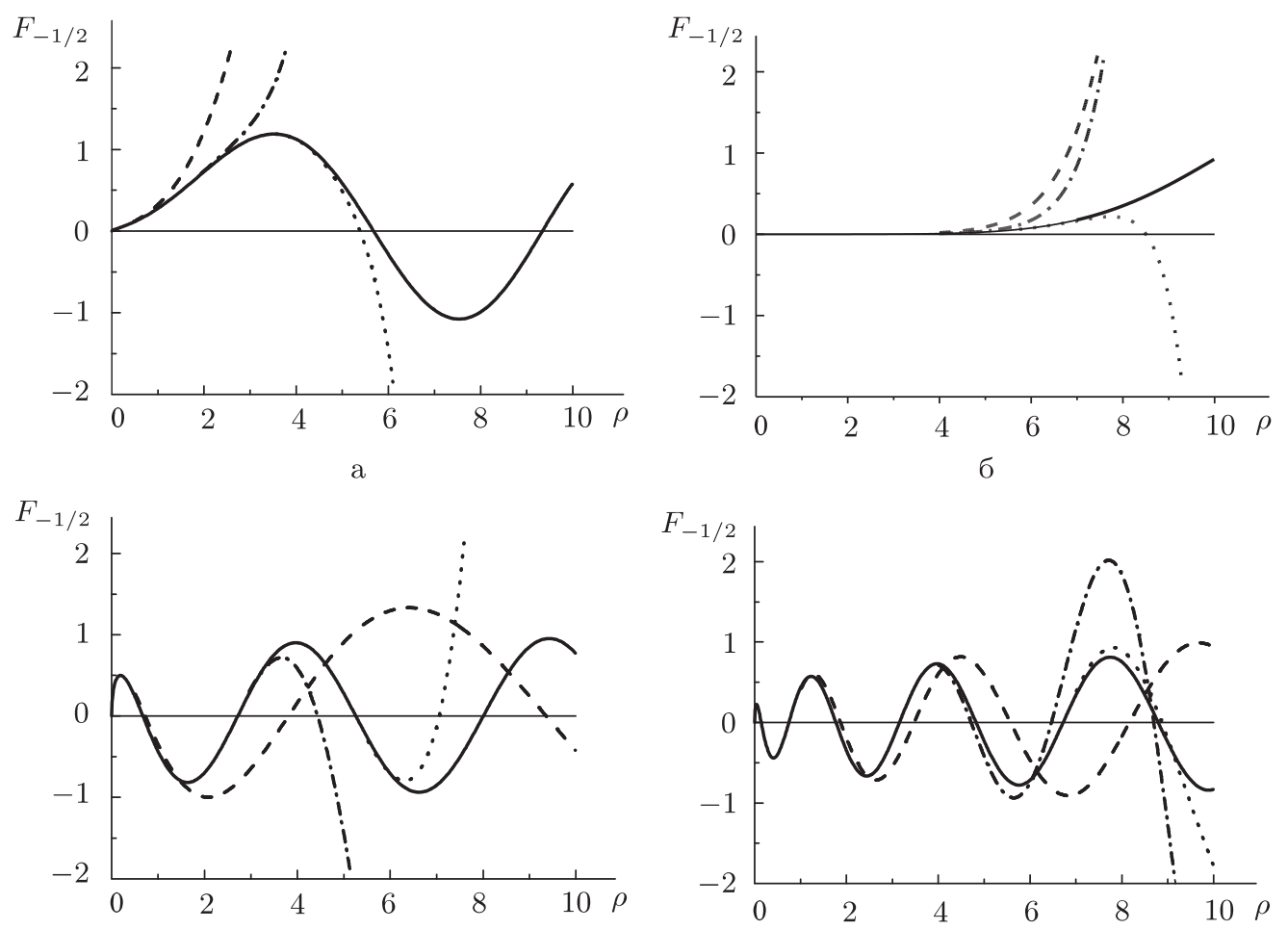

B

$\Gamma$

Рис. 1. График функции $F_{-1 / 2}(\rho, \alpha|\eta|)$ - сплошная кривая и графики функций $F_{-1 / 2, m}(\rho, \alpha|\eta|)$ для $m=0, m=2$ и $m=5$ - соответственно штриховая, штрихпунктирная и пунктирная кривые. Выбраны следующие значения параметров: $\alpha=1, q=0.5$ (а); $\alpha=1, q=0.1$ (б); $\alpha=-1, q=0.5$ (в) и $\alpha=-1$, $q=0.1($ г).

заданных формулами (28). На рис. 2 представлены графики функции $D_{\lambda m}$, рассчитанные по формулам (41) при $m=0,2,5$ и малом значении $\varepsilon=10^{-12}$. Согласно рис. 1 и рис. 2 абсолютная и относительная точности аппроксимации (40) соответственно на отрезке $0 \leqslant \rho \leqslant 10$ и на отрезке $0 \leqslant \rho \leqslant 5$ улучшаются с ростом $m$ при фиксированном значении переменной $q$ и с уменьшением значения этой переменной при данном $m$.

Уместным представляется следующее замечание. При выводе неравенств (37) и (39) все коэффициенты $b_{n s}$ заменялись наибольшим коэффициентом $b_{n, 2 n}$, а для функций Бесселя $J_{\mu}$ и $I_{\mu}$ использовались оценки (34) и (35), которые становятся излишне завышенными при больших значениях аргумента $v$. Поэтому тем же недостатком обладают и правые части мажорантных оценок (37) и (39) скорости сходимости рядов (31) и (38). Этот вывод также подтверждают результаты численного анализа, указывающие на сходимость этих рядов по числу $m$ их слагаемых не только при малых, но и при весьма больших значениях аргумента $\rho$. 

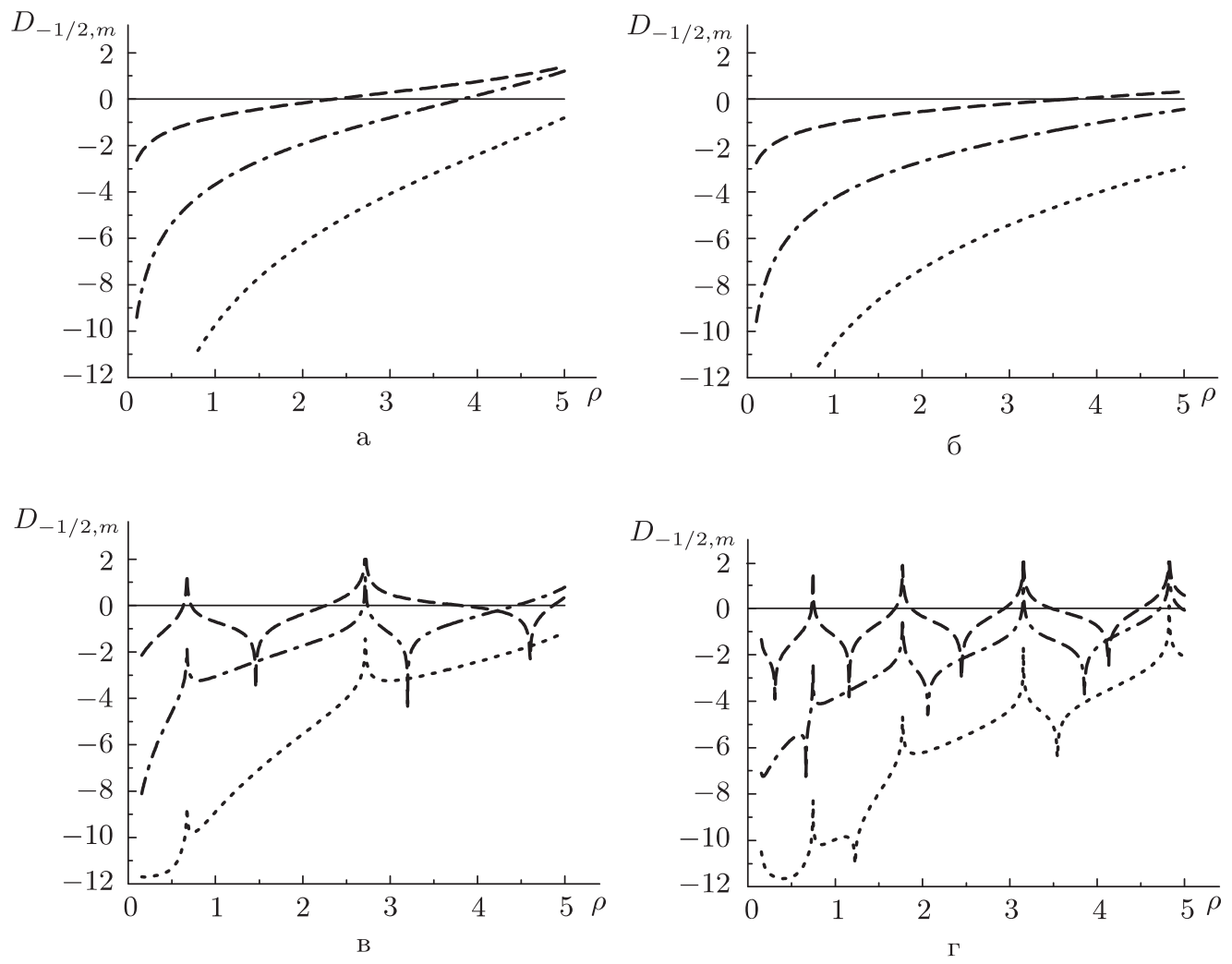

Рис. 2. Графики функции $D_{-1 / 2, m}(\rho, \alpha|\eta|)$ для $m=0, m=2$ и $m=5$ - соответственно штриховая, штрихпунктирная и пунктирная кривые. Различные рисунки отвечают тем же различным значениям параметров $\alpha$ и $q$, что и на рис. 1.

Кроме установленной выше равномерной сходимости, разложение (38) функции Кулона $F_{\lambda}$ полуцелого порядка по функциям Бесселя обладает двумя особыми свойствами. Во-первых, согласно формулам (26) и (32) все коэффициенты $b_{n s}$ этого разложения зависят только от их номеров $n, s$ и порядка $\lambda$. Во-вторых, зависимость любого слагаемого обсуждаемого разложения от переменной $q$ и от переменной $v$ выделяется в явном виде и представляется произведением четной степени аргумента $q$ и соответствующей функции Бесселя аргумента $v$. Известные разложения [17] функций Кулона $F_{\ell}(\rho, \alpha|\eta|)$ целого порядка $\ell=0,1, \ldots$ по функциям Бесселя-Клиффорда в случае $\alpha=1$ или по сферическим функциям Бесселя в случае $\alpha=-1$ не обладают аналогичными свойствами, потому что коэффициенты $b_{k}$ таких разложений зависят от переменной $\eta$, но в явном виде эта зависимость до сих пор не выписана.

Если $2 \lambda \geqslant-1$, то для исходного уравнения Кулона (5) точка $\rho=0$ является регулярной. Поэтому все без исключения представления (8)-(10) и (38) регулярного в этой точке решения $F_{\lambda}$ такого уравнения остаются в силе при любых вещественных $\lambda \geqslant-1 / 2$, в том числе и в случае $\lambda=\ell$, где $\ell$ - целое число. 
Следует отметить, что в частном случае $\alpha=1>0$ и $\lambda=\ell=0$ сумма слагаемых разложения (38) с номерами $n=0,1,2$ приобретает вид давно известного приближения, предложенного Фрёбергом в работе [20].

\section{4. НИЗКОЭНЕРГЕТИЧЕСКИЕ АСИМПТОТИКИ НЕРЕГУЛЯРНЫХ ФУНКЦИЙ КУЛОНА}

В настоящем разделе исследования выполняются по той же схеме, что и в предыдущем, но объектами исследования являются нерегулярные в точке $\rho=0$ решения уравнения Кулона $(5)$ - функции $G_{\lambda}(\rho, \eta)$ и $\Theta_{\lambda}(\rho, \eta)$. В п. 4.1 приводятся определения [11] и известные разложения [12] этих функций в бесконечные ряды, содержащие целые степени переменной $v$ и ее логарифм. Пункт 4.2 целиком посвящен выводам и пояснениям вспомогательных формул. В п. 4.3 эти формулы используются для представления функции $\Theta_{\lambda}$ в виде ряда Маклорена по переменной $q$ с остаточным слагаемым $O\left(q^{4}\right)$. В п. 4.4 для функции $\Theta_{\lambda}$ выводится формальное разложение по функциям Бесселя первого и второго рода.

4.1. Представления и свойства нерегулярных функций Кулона. Следуя работе [11], нерегулярной вещественной волновой функцией Кулона полуцелого порядка $\lambda=-1 / 2,1 / 2, \ldots$ мы считаем решение $G_{\lambda}(\rho, \eta)$ уравнения $(5)$, удовлетворяющее граничным условиям

$$
\begin{aligned}
& G_{\lambda}(\rho, \eta)= \begin{cases}O(\sqrt{\rho} \ln \rho), & 2 \lambda=-1, \\
O\left(\rho^{-\lambda}\right), & 2 \lambda>-1,\end{cases} \\
& G_{\lambda}(\rho, \eta)=\cos \left[\rho-\eta \ln (2 \rho)-\pi \frac{\lambda}{2}+\delta_{\lambda}^{\mathrm{c}}(\eta)\right]+O\left(\frac{1}{\rho}\right),
\end{aligned}
$$

Функцию $G_{\lambda}$ можно представить в виде суммы [12]:

$$
G_{\lambda}(\rho, \eta)=-\frac{h^{\mathrm{c}}(\eta)}{C_{-1 / 2}^{2}(\eta)} F_{\lambda}(\rho, \eta)+\frac{1}{q^{\lambda} C_{\lambda}(\eta)} \Theta_{\lambda}(\rho, \eta) .
$$

Эта сумма содержит неаналитическую функцию $h^{\mathrm{c}} \equiv \operatorname{Re} \Psi(1 / 2+i \eta)-\ln |\eta|$ и функцию $\Theta_{\lambda}$, которую можно записать в виде произведения,

$$
\Theta_{\lambda}(\rho, \pm|\eta|)=\frac{v}{(2 \lambda+1) !} \theta_{\lambda}^{ \pm}(v, q), \quad \rho=q x=q \frac{v^{2}}{4}, \quad \eta=\frac{\alpha}{2 q} .
$$

В этом произведении множитель $\theta_{\lambda}^{ \pm}(v, q)$ есть ряд [12]

$$
\begin{aligned}
\theta_{\lambda}^{ \pm}(v, q) \equiv & -\frac{1}{2}[(2 \lambda+1) !]^{2} P_{\lambda}\left(q^{2}\right) e^{i q v^{2} / 4} \times \\
& \times\left(\frac{v}{2}\right)^{\nu} \sum_{n=0}^{\infty} \frac{1}{(\nu+n) ! n !}\left(\lambda+1 \pm \frac{i}{2 q}\right)_{n}\left(-2 i q \frac{v^{2}}{4}\right)^{n} \times \\
& \times\left[\sum_{p=0}^{n+\lambda-1 / 2} \frac{1}{n+\lambda-p \pm i / 2 q}-\Psi(n+1)-\Psi(\nu+n+1)+2 \ln \frac{v}{2}\right]+ \\
& +\frac{1}{2} e^{i q v^{2} / 4}\left(-\frac{2}{v}\right)^{\nu} \sum_{n=0}^{\nu-1} \frac{(\nu-n-1) !}{n !}\left(-\lambda \pm \frac{i}{2 q}\right)_{n}\left(2 i q \frac{v^{2}}{4}\right)^{n},
\end{aligned}
$$


где полином $P_{\lambda}$ степени $\lambda+1 / 2$ от переменой $q^{2}$ определяется равенством

$$
\begin{aligned}
P_{\lambda}\left(q^{2}\right) & =q^{2 \lambda+1}\left[\frac{C_{\lambda}(\eta)}{C_{-1 / 2}(\eta)}\right]^{2}=\frac{1}{[(2 \lambda+1) !]^{2}} \times \\
& \times\left\{1+\frac{2}{3} \lambda(\lambda+1)(2 \lambda+1) q^{2}\left[1+\frac{1}{15}(\lambda-1)(2 \lambda-1)(5 \lambda+6) q^{2}+\cdots\right]\right\} .
\end{aligned}
$$

В (44) последняя сумма полагается равной нулю в случае $2 \lambda=-1, \nu \equiv 2 \lambda+1=0$.

Перечислим важные свойства функции $\theta_{\lambda}^{ \pm}$. Вследствие представления (44) в пределе $v \rightarrow 0$ эта функция неограниченно возрастает при $q<\infty$ :

$$
\theta_{\lambda}^{ \pm}(v, q) \sim\left\{\begin{array}{ll}
-\ln \frac{v}{2}, & 2 \lambda=-1, \\
\left(\frac{2}{v}\right)^{2 \lambda+1} \frac{(2 \lambda) !}{2}, & 2 \lambda>-1,
\end{array} \quad v \rightarrow 0, \quad q<\infty\right.
$$

Так как функция $\Theta_{\lambda}$ удовлетворяет исходному уравнению Кулона (5) и связана с функцией $\theta_{\lambda}^{ \pm}$равенством (43), то функция $\theta_{\lambda}^{ \pm}$является нерегулярным решением $y_{\nu}^{ \pm}, \nu=2 \lambda+1$, уравнения (30). Действительно, функция $\theta_{\lambda}^{ \pm}$имеет расходящуюся асимптотику (46), а уравнение Кулона (5) сводится к уравнению (30) подстановкой

$$
u_{\lambda}(\rho, \pm|\eta|)=\Theta_{\lambda}(\rho, \pm|\eta|)=\frac{v}{(2 \lambda+1) !} \theta_{\lambda}^{ \pm}(v, q), \quad y_{\nu}^{ \pm}(v, q)=\theta_{\lambda}^{ \pm}(v, q)
$$

В отличие от функции $G_{\lambda}$ обе функции $\Theta_{\lambda}$ и $\theta_{\lambda}^{ \pm}$являются четными функциями волнового числа $q$. Это свойство и вспомогательные соотношения, перечисленные в следующем пункте, окажутся ключевыми в п. 4.3 и 4.4 .

4.2. Вспомогательные соотношения. Разложение (44) функции $\theta_{\lambda}^{ \pm}$содержит уже исследованное в п. 3.2 произведение функций $(\lambda+1+i \alpha / 2 q)_{n}$ и $\left(-2 i q v^{2} / 4\right)^{n}$ и произведение функций $(-\lambda+i \alpha / 2 q)_{n}$ и $\left(2 i q v^{2} / 4\right)^{n}$. Построим ряд Маклорена для последнего произведения. Для этого используем определение $(13)$ полинома $A_{n}(t ; \lambda)$ по переменной $t=-2 i \alpha q$ и равенства

$$
\begin{aligned}
& \left(-\lambda+\frac{i \alpha}{2 q}\right)_{n}\left(2 i q \frac{v^{2}}{4}\right)^{n}=\left(-\alpha \frac{v^{2}}{4}\right)^{n} \prod_{j=1}^{n}[(-\lambda-1) t+j]= \\
& =\left(-\alpha \frac{v^{2}}{4}\right)^{n} A_{n}(t ;-\lambda-1)=\left(-\alpha \frac{v^{2}}{4}\right)^{n}\left(1+\sum_{p=1}^{4} a_{p} q^{p}+\cdots\right) .
\end{aligned}
$$

Эти равенства содержат коэффициенты $a_{p}$ полинома $A_{n}(t ;-\lambda-1)$. Представления таких коэффициентов через номер $n$ и индекс $\lambda$ можно получить из формул (18) для коэффициентов $a_{p}$ полинома $A_{n}(t ; \lambda)$ заменой $\lambda \mapsto-\lambda-1$, при которой сумма $a=n+(2 \lambda+1)$ перейдет в разность $a=n-(2 \lambda+1)$. 
В разложении (44) функции $\theta_{\lambda}^{ \pm}$имеется конечная сумма $\widetilde{S}_{n}(q ; \lambda)$ по индексу $p$. Покажем, что эту сумму можно представить в следующем виде:

$$
\begin{aligned}
& \widetilde{S}_{n}(q ; \lambda, \alpha) \equiv \sum_{p=0}^{n+\lambda-1 / 2} \frac{1}{n+\lambda-p+i \alpha / 2 q}= \\
& \quad=-i \alpha(2 n+\nu) q+\frac{1}{2}(2 n+\nu)^{2} q^{2}-\sum_{m=3}^{\infty}\left[\sum_{p=0}^{n+\lambda-1 / 2}(n+\lambda-p)^{m-1}\right](2 i \alpha q)^{m} .
\end{aligned}
$$

Введем переменную $t \equiv-2 i \alpha q$. В терминах этой переменной функция $\widetilde{S}_{n}$ записывается как

$$
\widetilde{S}_{n}(q ; \lambda, \alpha)=\sum_{p=0}^{n+\lambda-1 / 2} \frac{t}{n+\lambda-p+t},
$$

поэтому имеют место равенства

$$
\partial_{t}^{m} \widetilde{S}_{n}(q ; \lambda, \alpha)=(-1)^{m+1} m ! \sum_{p=0}^{n+\lambda-1 / 2} \frac{(n+\lambda-p)^{m-1}}{[(n+\lambda-p) t+1]^{m+1}}, \quad m \geqslant 1 .
$$

Благодаря этим равенствам в точке $t=0$ исследуемая функция $\widetilde{S}_{n}$ и все ее производные $\partial_{t}^{m} \widetilde{S}_{n}$ для $m \leqslant 4$ сводятся к табличным суммам (16). Используя их, построим ряд Маклорена для функции $\widetilde{S}_{n}$ по переменной $t$, а затем перейдем к переменной $q=i \alpha t / 2$. В итоге получим формулу (48), которую и требовалось доказать.

Напомним известные соотношения [16]: свойство пси-функции

$$
\Psi(t+n)=\sum_{p=1}^{n} \frac{1}{t+p-1}+\Psi(t)
$$

и разложения нерегулярных функций Бесселя $K_{\mu}(v)$ и $Y_{\mu}(v)$ целого порядка $\mu$

$$
\begin{aligned}
&\left\{\begin{array}{c}
2(-1)^{\mu} K_{\mu}(v) \\
-\pi Y_{\mu}(v)
\end{array}\right\}=\left(\mp \frac{v}{2}\right)^{\mu} \sum_{n=0}^{\infty}[\Psi(n+1)+\Psi(\nu+n+1)] \frac{\left( \pm v^{2} / 4\right)^{n}}{(\nu+n) ! n !}- \\
&-2 \ln \frac{v}{2}\left\{\begin{array}{l}
I_{\mu}(v) \\
J_{\mu}(v)
\end{array}\right\}+\left(\frac{2}{v}\right)^{\mu} \sum_{n=0}^{\mu-1} \frac{(\mu-n-1) !}{n !}\left( \pm \frac{v^{2}}{4}\right)^{n}
\end{aligned}
$$

При $\mu=0$ конечные суммы в этих разложениях полагаются равными нулю.

4.3. Ряды Маклорена для нерегулярных функций Кулона. Перейдем к выводу разложения функции $\theta_{\lambda}^{ \pm}$, заданной формулой (44), в ряд Маклорена по переменной $q$. Согласно этой формуле функция $\theta_{\lambda}^{ \pm}$содержит бесконечный ряд $S_{1}$ и конечную сумму $S_{2}$. Модуль суммы $S_{2}$ неограниченно возрастает только в случае $v \rightarrow+0$. Поэтому далее придется положить $v \neq 0$. Все производные суммы $S_{2}$ по переменной $q$ непрерывны при $q<\infty$. Способом, изложенным в п. 3.2 , нетрудно показать, что таким же свойством обладают и производные $\partial_{q}^{m} S_{1}$ ряда $S_{1}$ порядка $m \leqslant 4$. Следовательно, функцию $\theta_{\lambda}^{ \pm}$можно представить рядом Маклорена по переменной $q$ с остаточным слагаемым $R_{\lambda m}=O\left(q^{m}\right)$ при $m=4$ в области $v \in(0, \infty)$, $q \in[0, \infty)$. Осталось построить такой ряд. 
Предлагаемое построение принципиально ничем не отличается от подробно изложенного в п. 3.3 вывода разложений $(24)-(26)$ для функции $f_{\lambda}^{ \pm}$. Поэтому ограничимся только перечислением основных преобразований.

При любом $\lambda$ в качестве исходного используем разложение (44) функции $\theta_{\lambda}^{ \pm}$. Заменим в нем экспоненциальную функцию ее рядом Маклорена (23). Затем применим формулы (19) и (47), используем равенство (48) и свойство пси-функции (49). В полученном разложении выделим слагаемое, не зависящее от $q$, и слагаемые, пропорциональные $q^{2}$ и $q^{4}$. Бесконечные ряды по переменной $v$, содержащиеся в таких слагаемых, просуммируем в функции Бесселя первого и второго рода. Для этого используем соответствующие ряды $(21)$ и (50) и определения $(26)$ коэффициентов $b_{n s}$. В результате получим искомое разложение функции $\theta_{\lambda}^{ \pm}(v, q)$ в ряд Маклорена при $q \in[0, \infty)$ и $v \in(0, \infty)$ :

$$
\begin{aligned}
\theta_{\lambda}^{ \pm}(v, q)= & \sum_{n=0}^{1}\left(-\frac{q^{2}}{8}\right)^{n} \frac{1}{n !} \sum_{s=2 n}^{3 n}(-1)^{s} b_{n s} v^{s}\left\{\begin{array}{c}
K_{\nu+s}(v) \\
(-\pi / 2) Y_{\nu+s}(v)
\end{array}\right\}+ \\
& +\frac{2}{3} \lambda(\lambda+1)(2 \lambda+1) q^{2}\left\{\begin{array}{c}
K_{\nu}(v) \\
(-\pi / 2) Y_{\nu}(v)
\end{array}\right\}-\frac{q^{2}}{12} b_{00}\left\{\begin{array}{l}
I_{\nu}(v) \\
J_{\nu}(v)
\end{array}\right\}+R_{\lambda 4}(v, q)
\end{aligned}
$$

с остаточным слагаемым $R_{\lambda 4}=O\left(q^{4}\right)$.

Данный ряд в отличие от ряда Маклорена $(24)-(26)$ для функции $f_{\lambda}^{ \pm}$содержит нерегулярные функции Бесселя наряду с регулярными. Поясним, почему это так. В исходном представлении (44) функции $\theta_{\lambda}^{ \pm}$имеется конечная сумма по индексу $p$ и алгебраическая сумма двух пси-функций и логарифмической функции. Первая и вторая из этих сумм при выводе ряда Маклорена (51) для функции $\theta_{\lambda}^{ \pm}$порождают соответственно разложения (21) и (50) регулярных и нерегулярных функций Бесселя.

Ряды Маклорена для функций $G_{\lambda}$ и $\Theta_{\lambda}$ с остаточными слагаемыми $O\left(q^{4}\right)$ построить нетрудно. Для этого нужно в правых частях равенств (42) и (43) заменить функции $F_{\lambda}$ и $\theta_{\lambda}^{ \pm}$соответствующими суммами $(27),(28)$ и (51).

\section{4. Разложения нерегулярных функций Кулона по функция Бесселя.} Наша следующая задача - представить функцию $\theta_{\lambda}^{ \pm}(v, q)$ в виде такого бесконечного ряда по четным степеням $q^{2 n}$ аргумента $q$, у которого частичная сумма порядка $n=2$ совпадает с правой частью равенства (51). Решение этой задачи начнем со следующих предположений. Пусть все коэффициенты $b_{n s}$ подчинены цепочке рекуррентных уравнений (32), а ряд

$$
g_{\lambda}^{ \pm}(v, q) \equiv \sum_{n=0}^{\infty}\left(-\frac{q^{2}}{8}\right)^{n} \frac{1}{n !} \sum_{s=2 n}^{3 n}(-1)^{s} b_{n s} v^{s}\left\{\begin{array}{c}
K_{\nu+s}(v) \\
(-\pi / 2) Y_{\nu+s}(v)
\end{array}\right\}
$$

равномерно сходится при $0 \leqslant q<\infty$ и $0<v<\infty$.

Выявим основные свойства этого ряда. Сначала найдем старшее слагаемое его асимптотики в пределе $v \rightarrow 0$. Для этого в представлении (52) заменим все функции Бесселя старшими слагаемыми их разложений (50) и в случае $2 \lambda=-1$ получим формулу

$$
g_{\lambda}^{ \pm}(v, q)=-\ln \frac{v}{2}+\frac{1}{2} \sum_{n=0}^{\infty}\left(-\frac{q^{2}}{8}\right)^{n} \frac{1}{n !} \sum_{s=2 n}^{3 n}(-2)^{s} b_{n s}+O\left(v^{2} \ln v\right), \quad v \rightarrow 0,
$$


а в случае $2 \lambda \geqslant 1-$ формулу

$$
g_{\lambda}^{ \pm}(v, q)=2\left(\frac{2}{v}\right)^{\nu} \sum_{n=0}^{\infty}\left(-\frac{q^{2}}{8}\right)^{n} \frac{1}{n !} \sum_{s=2 n}^{3 n}(-2)^{s} b_{n s}(\nu+s-1) !+O\left(v^{-\nu+1}\right), \quad v \rightarrow 0 .
$$

Теперь в уравнении (30) заменим функцию $y_{\nu}^{ \pm}$рядом $g_{\lambda}^{ \pm}$. Используя для функций Бесселя $K_{\mu}$ и $Y_{\mu}$ и их производных известные рекуррентные соотношения [16], убедимся в том, что ряд $g_{\lambda}^{ \pm}$является решением уравнения (30). Согласно асимптотикам (53) и (54) это решение нерегулярно в точке $v=0$ и поэтому линейно не зависит от уже исследованного в п. 3.3-3.5 регулярного решения $f_{\lambda}^{ \pm}$уравнения (30).

Перейдем к построению решения $\theta_{\lambda}^{ \pm}$уравнения (30) в виде разложения по функциям Бесселя. Это уравнение имеет два линейно независимых решения $f_{\lambda}^{ \pm}$и $g_{\lambda}^{ \pm}$, и любое другое решение можно представить в виде линейной комбинации этих решений с коэффициентами $c_{\lambda}^{ \pm}$и $d_{\lambda}^{ \pm}$, которые в общем случае зависят от $q$. Представим искомое решение $\theta_{\lambda}^{ \pm}$в виде такой комбинации,

$$
\theta_{\lambda}^{ \pm}(v, q)=c_{\lambda}^{ \pm}(q) f_{\lambda}^{ \pm}(v, q)+d_{\lambda}^{ \pm}(q) g_{\lambda}^{ \pm}(v, q)
$$

и приступим к определению коэффициентов $c_{\lambda}^{ \pm}$и $d_{\lambda}^{ \pm}$.

Сначала найдем функцию $d_{\lambda}^{ \pm}(q)$. Для этого применим самый простой способ: в соотношении (55) перейдем к пределу $v \rightarrow 0, q<\infty$. В этом пределе согласно представлению (10) функция $f_{\lambda}^{ \pm}$ограничена, а функции $\theta_{\lambda}^{ \pm}$и $g_{\lambda}^{ \pm}$неограниченно возрастают в соответствии с формулами (46) и $(53)$, (54). Поэтому в обсуждаемом пределе соотношение (55) вырождается в уравнение относительно неизвестной функции $d_{\lambda}^{ \pm}$. Это уравнение не содержит функции $c_{\lambda}^{ \pm}$и имеет единственное решение:

$$
\begin{aligned}
& d_{\lambda}^{ \pm}(q) \equiv 1, \quad 2 \lambda=-1 \\
& d_{\lambda}^{ \pm}(q)=\left\{\sum_{n=0}^{\infty}\left(-\frac{q^{2}}{8}\right)^{n} \frac{1}{n !} \sum_{s=2 n}^{3 n}(-2)^{s} b_{n s} \frac{(2 \lambda+s) !}{(2 \lambda) !}\right\}^{-1}, \quad 2 \lambda>-1 .
\end{aligned}
$$

Теперь найдем функцию $c_{\lambda}^{ \pm}$. Согласно формулам $(21),(31),(44),(50)$ и (52) левая и правая части соотношения (55) являются бесконечными разложениями по системе линейно независимых функций $v^{n}, v^{m} \ln v$ с целыми номерами $n$ и $m$. Следовательно, это соотношение верно тогда и только тогда, когда каждое слагаемое $s_{n}(q) v^{n}$ или $t_{n}(q) v^{m} \ln v$ разложения (44) функции $\theta_{\lambda}^{ \pm}$равно соответствующему слагаемому $\tilde{s}_{n}(q) v^{n}$ или $\tilde{t}_{n}(q) v^{m} \ln v$ разложения функции $c_{\lambda}^{ \pm} f^{ \pm}+d_{\lambda}^{ \pm} g^{ \pm}$. В частности, должно выполняться равенство $s_{n}(q) v^{n}=\tilde{s}_{n}(q) v^{n}$ с номером $n=\nu$. Из этого условия следует представление функции $c_{\lambda}^{ \pm}$через уже найденную функцию $d_{\lambda}^{ \pm}$: в случае $2 \lambda=-1$ имеем

$$
c_{\lambda}^{ \pm}(q)=-\frac{1}{2} \sum_{n=1}^{\infty}\left(-\frac{q^{2}}{8}\right)^{n} \frac{1}{n !} \sum_{s=2 n}^{3 n}(-2)^{s} b_{n s}(s-1) !,
$$

а в случае $2 \lambda>-1$

$$
\begin{aligned}
2 c_{\lambda}^{ \pm}(q)= & {[(2 \lambda+1) !]^{2}\left[P_{\lambda}\left(q^{2}\right)-d_{\lambda}^{ \pm}(q)\right][\Psi(1)+\Psi(2 \lambda+2)]-} \\
& -[(2 \lambda+1) !]^{2} P_{\lambda}\left(q^{2}\right) \sum_{p=0}^{\lambda-1 / 2} \frac{1}{\lambda-p \pm i / 2 q}+(i q)^{2 \lambda+1}(2 \lambda) ! \sum_{n=0}^{2 \lambda} \frac{2^{n}}{n !}\left(-\lambda \pm \frac{i}{2 q}\right)_{n} .
\end{aligned}
$$


Обсудим теперь свойства функций $c_{\lambda}^{ \pm}(q)$ и $d_{\lambda}^{ \pm}(q)$. Согласно полученным выше представлениям эти функции являются четными, удовлетворяют равенствам $c_{\lambda}^{+}(q)=c_{\lambda}^{-}(q), d_{\lambda}^{+}(q)=d_{\lambda}^{-}(q)$ и имеют следующие асимптотики:

$$
c_{\lambda}^{ \pm}(q)=-\frac{q^{2}}{12}+O\left(q^{2}\right), \quad d_{\lambda}^{ \pm}(q)=1+\frac{2}{3} \lambda(\lambda+1)(2 \lambda+1) q^{2}+O\left(q^{4}\right), \quad q \rightarrow+0 .
$$

Заметим, что благодаря таким асимптотикам и определению (45) полинома $P_{\lambda}$ из представления (55) функции $\theta_{\lambda}$ следует ее ряд Маклорена (51). Тем самым мы решили задачу, поставленную в начале настоящего пункта.

Теперь выведем низкоэнергетическую асимптотику нерегулярной функции Кулона $\Theta_{\lambda}$, заданную рядом (44). Символами $f_{\lambda m}^{ \pm}$и $g_{\lambda m}^{ \pm}$обозначим частичные суммы порядка $m$ для бесконечных рядов (31) и (52). Введем функции $\theta_{\lambda m}^{ \pm}$и $\Theta_{\lambda m}$ соотношениями

$$
\theta_{\lambda m}(v, q) \equiv c_{\lambda}(q) f_{\lambda m}^{ \pm}(v, q)+d_{\lambda}(q) g_{\lambda m}^{ \pm}(v, q), \quad \Theta_{\lambda m}(\rho, \pm|\eta|) \equiv \frac{v}{(2 \lambda+1) !} \theta_{\lambda m}^{ \pm}(v, q) .
$$

Используя эти определения, запишем нерегулярную функцию Кулона $\Theta_{\lambda}$ в низкоэнергетическом пределе как

$$
\Theta_{\lambda}(\rho, \pm|\eta|)=\Theta_{\lambda m}(\rho, \pm|\eta|)+O\left(q^{2 m+2}\right), \quad q \rightarrow+0, \quad 0<v<\infty
$$

где первое слагаемое задано формулой

$$
\begin{aligned}
\Theta_{\lambda m}(\rho, \pm|\eta|)= & \frac{v}{(2 \lambda+1) !} \sum_{n=0}^{m}\left(-\frac{q^{2}}{8}\right)^{n} \frac{1}{n !} \times \\
& \times \sum_{s=2 n}^{3 n}(-1)^{s} b_{n s} v^{s}\left\{\begin{array}{c}
c_{\lambda}^{+}(q) I_{\nu+s}(v)+d_{\lambda}^{+}(q) K_{\nu+s}(v) \\
(-1)^{s} c_{\lambda}^{-}(q) J_{\nu+s}(v)+d_{\lambda}^{-}(q)(-\pi / 2) Y_{\nu+s}(v)
\end{array}\right\} .
\end{aligned}
$$

Сформулируем основной результат настоящего пункта: если ряд (52) сходится равномерно, то функция $\Theta_{\lambda m}, m>2$, является низкоэнергетической асимптотикой нерегулярной функции Кулона $\Theta_{\lambda}$.

Доказать равномерную сходимость этого ряда не удалось из-за отсутствия в справочной литературе [16]-[18] равномерных по аргументу $v$ мажорантных оценок функций $Y_{\mu}(v)$ и $K_{\mu}(v)$. Итоги численного сравнения функций $\Theta_{\lambda}, 2 \lambda= \pm 1,3$, с функциями $\Theta_{\lambda m}, m=0,5,10$, проведенного по методике, изложенной в п. 3.5, подтверждают справедливость приближения $\Theta_{\lambda} \approx \Theta_{\lambda m}$ при $0<v<\infty$ и $q \rightarrow+0$ и указывают на улучшение точности этого приближения при увеличении числа $m$.

Следует отметить, что предложенный в настоящем пункте способ разложения нерегулярных функций Кулона $\Theta_{\lambda}$ полуцелого порядка $\lambda$ по функциям Бесселя примени́м и в случае целых значений $\lambda=\ell$, где $\ell=0,1, \ldots$.

\section{5. НИЗКОЭНЕРГЕТИЧЕСКАЯ АСИМПТОТИКА ВОЛНОВОЙ ФУНКЦИИ}

В работе [7] показано, что уравнение Шредингера (1) имеет точное решение

$$
\Psi^{\mathrm{c}}(\rho, \varphi, \eta)=C(\eta) e^{i \rho \cos \varphi} \Phi(-i \eta, 1 / 2, \xi), \quad \xi \equiv 2 i \rho \sin ^{2} \frac{\varphi}{2},
$$


где функция $C(\eta)$ определена равенством

$$
C(\eta)=\frac{\Gamma(1 / 2+i \eta)}{\Gamma(1 / 2)} e^{-\pi \eta / 2}
$$

Как пояснялось в работе [11], функция $\Psi^{\mathrm{c}}$, заданная формулами (59), (60), является физически приемлемой волновой функцией двумерного кулоновского рассеяния квантовой частицы неподвижным силовым центром. В п. 5.1 и 5.2 мы получим в явном виде асимптотику функции $\Psi^{\mathrm{c}}$ в пределе $q \rightarrow+0$, сохранив множители $C(\eta)$ и $e^{i \rho \cos \varphi}$. Понятно, что для решения такой задачи достаточно заменить в формуле (59) функцию Куммера ее рядом Маклорена по переменной $q$.

\section{1. Приближение волновой функции конечной суммой ряда Маклоре-}

на. Построим ряд Маклорена методом, предложенным в п. 3.2. Будем использовать переменную $w \equiv \sqrt{2 \xi / i q}$ и прежние обозначения функций Бесселя (21) первого рода $Z_{\mu}^{+} \equiv I_{\mu}, Z_{\mu}^{-} \equiv J_{\mu}$. Применив известные равенства [16]

$$
\left(\frac{1}{2}\right)_{n}=\frac{\Gamma(1 / 2+n)}{\Gamma(1 / 2)}=\frac{\Gamma(1 / 2+n)}{\sqrt{\pi}},
$$

получим для исследуемой функции Куммера представление в виде ряда:

$$
\Phi(-i \alpha|\eta|, 1 / 2 ; \xi)=\sqrt{\pi} \sum_{n=0}^{\infty} \frac{(-i \alpha / 2 q)_{n}}{\Gamma(1 / 2+n) n !}\left(2 i q \frac{w^{2}}{4}\right)^{n}, \quad \eta=\frac{\alpha}{2 q}, \quad \xi=i q \frac{w^{2}}{2}
$$

Каждое слагаемое этого ряда содержит произведение символа Похгаммера и четной степени переменной $w$. Если $t=-2 i \alpha q$, а $A_{n}(t ; \lambda)$ - полином $(13)$, то для такого произведения верна цепочка равенств

$$
\begin{aligned}
& \left(-\frac{i \alpha}{2 q}\right)_{n}\left(2 i q \frac{w^{2}}{4}\right)^{n}=\left(\alpha \frac{w^{2}}{4}\right)^{n} \prod_{j=1}^{n}[(j-1)(-t)+1]= \\
& =\left.\left(\alpha \frac{w^{2}}{4}\right)^{n} A_{n}(-t, \lambda)\right|_{\lambda=-1}=\left(\alpha \frac{w^{2}}{4}\right)^{n}\left[1+\sum_{j=1}^{4} a_{j} q^{j}+\cdots+(n-1) !(2 i \alpha q)^{n-1}\right],
\end{aligned}
$$

в которой коэффициенты $a_{j}$ выражаются через номер $n$ и произведение $a=n(n-1)$ с помощью следующих формул:

$$
\begin{gathered}
a_{1}=i \alpha a, \quad a_{2}=-\frac{a}{6}(n-2)(3 n-1), \quad a_{3}=-i \alpha \frac{a^{2}}{6}(n-2)(n-3), \\
a_{4}=\frac{a}{360}(n-2)(n-3)(n-4)\left(15 n^{3}-30 n^{2}+5 n+2\right) .
\end{gathered}
$$

Действительно, полином $\left.A_{n}(-t ; \lambda)\right|_{\lambda=-1}$ получается из полинома $A_{n}(t ; \lambda)$ заменой $\alpha \mapsto-\alpha$ и $\lambda \mapsto-1$, а после такой замены коэффициенты (18) полинома $A_{n}(t ; \lambda)$ становятся коэффициентами $(63)$ полинома $\left.A_{n}(-t ; \lambda)\right|_{\lambda=-1}$. 
Теперь преобразуем ряд (61). Используем равенства (20) с индексом $\nu=-1 / 2$, разложения функций Бесселя $Z_{\mu}^{ \pm}$в степенные ряды (21) и формулы (62), (63). В результате получим искомый ряд Маклорена:

$$
\begin{aligned}
& \Phi(\mp i|\eta|, 1 / 2 ; \xi)=\sqrt{\pi}\left(\frac{w}{2}\right)^{1 / 2}\left\{Z_{-1 / 2}^{ \pm}(w) \pm\left(i \frac{q}{2}\right) \frac{w}{2} Z_{3 / 2}^{ \pm}(w)+\right. \\
& +\left(i \frac{q}{2}\right)^{2} w^{3}\left[ \pm \frac{2}{3} Z_{5 / 2}^{ \pm}(w)+\frac{w}{8} Z_{7 / 2}^{ \pm}(w)\right]- \\
& \left.-\left(i \frac{q}{2}\right)^{3} w^{4}\left[ \pm Z_{7 / 2}^{ \pm}(w)+\frac{w}{3} Z_{9 / 2}^{ \pm}(w) \pm \frac{w^{2}}{48} Z_{11 / 2}^{ \pm}(w)\right]+R_{4}^{ \pm}(w, q)\right\} .
\end{aligned}
$$

Согласно выводам, сформулированным в конце п. 3.2 , остаток $R_{4}^{ \pm}$этого ряда убывает, как $O\left(q^{4}\right)$, если $q \rightarrow+0$ и $w<\infty$.

При тех же условиях формула, полученная заменой в произведении (59) функции Куммера правой частью равенства (63), есть низкоэнергетическая асимптотика волновой функции $\Psi^{\mathrm{c}}$. Обсудим физически интересные следствия такой асимптотики.

Первое следствие заключается в том, что правая часть равенства

$$
\lim _{q \rightarrow+0} \frac{\Psi^{\mathrm{c}}(\rho, \varphi, \pm|\eta|)}{C(\eta) e^{i \rho \cos \varphi}}=\sqrt{\pi}\left(\frac{w}{2}\right)^{1 / 2} Z_{-1 / 2}^{ \pm}(w)
$$

является точным и физически приемлемым решением уравнения Шредингера (1) при нулевой энергии квантовой частицы $p_{1}$.

Используя определение (60) функции $C(\eta)$ и предельное соотношение (65), выведем еще два следствия: в точке $r=0$ волновая функция $\Psi^{\text {c }}$ удовлетворяет равенствам

$$
\Psi^{\mathrm{c}}(\rho, \varphi, \eta)=C(\eta), \quad\left|\Psi^{\mathrm{c}}(\rho, \varphi, \eta)\right|^{2}=C^{2}(\eta)=\frac{e^{-\pi \eta}}{\operatorname{ch}(\pi \eta)}
$$

эти равенства порождают следующие приближения при $r=0$ и $q \ll 1$ :

$$
\left|\Psi^{\mathrm{c}}(\rho, \varphi, \pm|\eta|)\right|^{2} \approx 2\left\{\begin{array}{l}
e^{-2 \pi \eta} \\
1
\end{array}\right.
$$

Полученные приближения означают, что в пределе нулевой энергии в точке $r=0$ квадрат модуля волновой функции $\Psi^{\mathrm{c}}$ квантовой частицы $p_{1}$ быстро сходится к нулю в случае кулоновского отталкивания и имеет конечный ненулевой предел, равный двум, в случае кулоновского притяжения.

5.2. Разложение волновой функции по функциям Бесселя. Функция Куммера $\Phi(-i \eta, 1 / 2 ; \xi)$ является регулярным решением следующего гипергеометрического уравнения [16]:

$$
\left[\xi \partial_{\xi}^{2}+\left(\frac{1}{2}-\xi\right) \partial_{\xi}+i \eta\right] \Phi(-i \eta, 1 / 2 ; \xi)=0 .
$$

Построим формальное разложение этого решения по функциям Бесселя $Z_{\mu}^{ \pm}(w)$ apгумента $w \equiv \sqrt{2 \xi / i q}$ и полуцелого порядка $\mu \geqslant-1 / 2$. Для этого подстановкой

$$
\Phi(\mp i|\eta|, 1 / 2 ; \xi)=\sqrt{\pi}\left(\frac{w}{2}\right)^{1 / 2} \phi^{ \pm}(w, q), \quad \xi=i q \frac{w^{2}}{2}
$$


сведем упомянутое выше гипергеометрическое уравнение к уравнению

$$
\left[\partial_{w}^{2}+\frac{1}{w} \partial_{w} \mp 1-\frac{1}{4 w^{2}}\right] \phi^{ \pm}(w, q)-i \frac{q}{2}\left(2 w \partial_{w}+1\right) \phi^{ \pm}(w, q)=0,
$$

которому при $q=0$ удовлетворяет функция Бесселя $Z_{-1 / 2}^{ \pm}(w)$.

Далее, используя известные рекуррентные соотношения для функций Бесселя [17], убедимся в том, что уравнение (67) имеет формальное решение

$$
\phi^{ \pm}(w, q)=Z_{-1 / 2}^{ \pm}(w)+\sum_{n=1}^{\infty}\left(i \frac{q}{2}\right)^{n} \sum_{s=n+1}^{2 n}( \pm 1)^{n+s} p_{n s} w^{s} Z_{s-1 / 2}^{ \pm}(w),
$$

если $p_{00}=1$, а все остальные коэффициенты $p_{n s}, n=1,2, \ldots$, подчиняются следующей цепочке уравнений:

$$
\begin{aligned}
(n+1) p_{n, s} & =2 n p_{n-1, s-1}, & & s=n+1 ; \\
s p_{n s} & =2(s-1) p_{n-1, s-1}+p_{n-1, s-2}, & & s=n+2, n+3, \ldots, 2 n-1 ; \\
2 n p_{n, s} & =p_{n-1, s-2}=0, & & s=2 n .
\end{aligned}
$$

Из этой цепочки следуют равенства

$$
p_{12}=\frac{1}{2}, \quad p_{23}=\frac{2}{3}, \quad p_{24}=\frac{1}{8}, \quad p_{34}=1, \quad p_{35}=\frac{1}{3}, \quad p_{36}=\frac{1}{48}
$$

и соотношения

$$
\frac{1}{2^{n} n !}=p_{n, 2 n}<p_{n, 2 n-1}<\cdots<p_{n, n+2}<p_{n, n+1}=\frac{2^{n-1}}{n+1}, \quad n=1,2, \ldots,
$$

согласно которым все коэффициенты $p_{n s}$ положительные и $p_{n, n+1}>p_{n s}$ при каждом фиксированном $n$ и при $s>n+1$.

Теперь, применив формулы (66) и (68), запишем искомое формальное разложение функции Куммера $\Phi(-i \eta, 1 / 2 ; \xi)$ по функциям Бесселя в следующем виде:

$$
\begin{gathered}
\Phi(\mp i \eta, 1 / 2 ; \xi)=\sqrt{\pi}\left(\frac{w}{2}\right)^{1 / 2}\left(Z_{-1 / 2}^{ \pm}(w)+T^{ \pm}(w, q)\right), \\
T^{ \pm}(w, q) \equiv \sum_{n=1}^{\infty}\left(i \frac{q}{2}\right)^{n} \sum_{s=n+1}^{2 n}( \pm 1)^{n+s} p_{n s} w^{s} Z_{s-1 / 2}^{ \pm}(w) .
\end{gathered}
$$

Отметим, что благодаря равенствам (69) частичная сумма полученного ряда (71), слагаемые которой содержат целые степени $q^{n}, n=0,1,2,3$, переменной $q$, совпадает с рядом Маклорена (63) функции $\Phi(\mp i \eta, 1 / 2 ; \xi)$.

Докажем следующее утверждение: если $0 \leqslant w<\infty$ и $0 \leqslant q<\infty$, то ряд $T^{ \pm}(w, q)$ сходится равномерно. Доказательство разобьем на три этапа.

Первый этап - построение мажоранты $T_{1}^{ \pm}(w, q)$ модуля ряда $T^{ \pm}(w, q)$. Для этого в $T^{ \pm}(w, q)$ заменим все функции $(i q / 2)^{n}$ и $Z_{s-1 / 2}^{ \pm}$их модулями, а затем выполним еще две замены. При каждом $n$ заменим все коэффициенты $p_{n s}$ наибольшим коэффициентом $p_{n, n+1}$, который согласно формулам (70) равен $2^{n-1} /(n+1)$. Модули всех функций Бесселя $Z_{\mu}^{-}(w)=J_{\mu}(w)$ и $Z_{\mu}^{+}(w)=I_{\mu}(w)$ для $\mu=s-1 / 2$ заменим 
правыми частями соответствующих оценок (34) и (35). Полученный ряд обозначим как $T_{1}^{ \pm}$. Согласно выполненным преобразованиям имеем

$$
\begin{gathered}
\left|T^{ \pm}(w, q)\right|<T_{1}^{ \pm}(w, q) \\
T_{1}^{ \pm}(w, q) \equiv\left\{\begin{array}{c}
\operatorname{ch} w \\
1
\end{array}\right\} \frac{1}{\sqrt{2 w}} \sum_{n=1}^{\infty} \frac{q^{n}}{n+1} \sum_{s=n+1}^{2 n}\left(\frac{w^{2}}{2}\right)^{2 s} \frac{1}{\Gamma(s+1 / 2)} .
\end{gathered}
$$

Второй этап - построение мажоранты $T_{2}^{ \pm}$ряда $T_{1}^{ \pm}$. На этом этапе ключевыми будут следующие известные свойства гамма-функции [17]:

$$
\Gamma(s-1)=(s-2) !<\Gamma(s+1 / 2)=\sqrt{\pi} 2^{-s}(2 s-1) ! !, \quad s=2,3, \ldots .
$$

Преобразуем ряд $T_{1}^{ \pm}$. Каждое число $\Gamma(s+1 / 2), s=n+1, n+2, \ldots, 2 n$, заменим меньшим числом $(s-2)$ !. Затем перейдем от индекса суммирования $s$ к индексу $m=s-n-1$. Наконец, воспользуемся неравенством $(m+n-1) ! \leqslant(n-1) ! m$ ! и известным разложением экспоненциальной функции в бесконечный ряд Маклорена [13]. В итоге из ряда $T_{1}$ получим мажорирующую его функцию $T_{2}$ :

$$
T_{1}^{ \pm}(w, q)<T_{2}^{ \pm}(w, q) \equiv\left\{\begin{array}{c}
\operatorname{ch} w \\
1
\end{array}\right\}\left(\frac{w}{2}\right)^{3 / 2}\left(1-e^{q w^{2} / 2}\right) e^{w^{2} / 2}
$$

Третий этап - мажорантная оценка исходного ряда $T^{ \pm}$, заданного в формуле (71). Согласно предыдущим этапам верны неравенства

$$
\left|T^{ \pm}(w, q)\right|<T_{1}^{ \pm}(w, q)<T_{2}^{ \pm}(w, q)
$$

а функция $T_{2}^{ \pm}$принимает конечные значения, если $0 \leqslant w \leqslant \infty$ и $0 \leqslant q \leqslant \infty$. Следовательно, при тех же условиях ряд $T^{ \pm}$сходится равномерно, что и требовалось доказать.

Символом $T_{m}^{ \pm}(w, q), m=1,2, \ldots, m<\infty$, обозначим частичную сумму ряда $T^{ \pm}$, содержащую все целые степени $q^{n}, n=1,2, \ldots, m$, аргумента $q$. Применив изложенный выше поэтапный способ, нетрудно доказать следующее асимптотическое соотношение:

$$
T^{ \pm}(w, q)=T_{m}^{ \pm}(w, q)+O\left(q^{m+1}\right), \quad q \rightarrow+0, \quad 0 \leqslant w<\infty .
$$

Завершим настоящий пункт выводом разложения волновой функции $\Psi^{\mathrm{c}}$ по функциям Бесселя и обсуждением ее низкоэнергетического приближения. Используя формулы (59) и (71), представим искомое разложение в следующем виде:

$$
\begin{aligned}
\Psi^{\mathrm{c}}(\rho, \varphi, \pm|\eta|)= & C(\eta) e^{i \rho \cos \varphi} \sqrt{\pi}\left(\frac{w}{2}\right)^{1 / 2} \times \\
& \times\left\{Z_{-1 / 2}^{ \pm}(w)+\sum_{n=1}^{\infty}\left(i \frac{q}{2}\right)^{n} \sum_{s=n+1}^{2 n}( \pm 1)^{n+s} p_{n s} w^{s} Z_{s-1 / 2}^{ \pm}(w)\right\}
\end{aligned}
$$

где $w=\sqrt{8|\eta| \rho} \sin (\varphi / 2)$. 
Пусть $\Psi_{m}^{\mathrm{c}}(\rho, \varphi, \pm|\eta|)$ - конечная $(m<\infty)$ сумма разложения (73), содержащая функции $q^{n}, n=0,1, \ldots, m$. Тогда благодаря свойству $(72)$ ряда $T^{ \pm}$верно равенство

$$
\Psi^{\mathrm{c}}(\rho, \varphi, \pm|\eta|)=\Psi_{m}^{\mathrm{c}}(\rho, \varphi, \pm|\eta|)+O\left(C(\eta) e^{i \rho \cos \varphi} q^{m+1}\right), \quad q \rightarrow+0, \quad 0 \leqslant \rho<\infty .
$$

Это равенство означает, что функция $\Psi_{m}^{\mathrm{c}}$ аппроксимирует волновую функцию $\Psi^{\mathrm{c}}$ в пределе низких энергий при $0 \leqslant \rho<\infty$.

\section{6. ЗАКЛЮЧЕНИЕ}

Просуммируем основные результаты настоящей работы, полученные впервые.

Для всех регулярных волновых функций $F_{\lambda}$ и $\Psi^{\mathrm{c}}$ двумерного кулоновского рассеяния квантовой частицы силовым центром выведены и исследованы разложения (38) и (73) по функциям Бесселя первого рода. Равномерная сходимость таких разложений доказана для $\rho \in[0, \infty), q \in[0, \infty)$. Показано, что конечные (порядка $m<\infty$ ) суммы $F_{\lambda m}$ и $\Psi_{m}^{\mathrm{c}}$ таких разложений являются низкоэнергетическими (при $q \rightarrow+0$ ) асимптотиками функций $F_{\lambda}$ и $\Psi^{\mathrm{c}}$ в области $\rho<\infty$.

Установлено, что низкоэнергетические асимптотики нерегулярных решений $\Theta_{\lambda}$ уравнения Кулона (5) при $\rho \in(0, \infty)$ определяются формулами $(43),(51)$. Для таких решений получены формальные разложения (58) по функциям Бесселя первого и второго рода. Сходимость этих разложений в области $\rho \in(0, \infty)$ подтверждена численным анализом.

Полученные представления (38), (51) и (73) волновых функций $F_{\lambda}, \Theta_{\lambda}$ и $\Psi^{\mathrm{c}}$ предлагается использовать для теоретической обработки результатов экспериментальных исследований [21] столкновений ультрахолодных атомов и ионов в магнитооптических ловушках дискообразной формы.

\section{Список литературы}

[1] H. Friedrich, Scattering Theory, Lecture Notes in Physics, 872, Springer, Berlin, 2013.

[2] B. Zaslow, M.E. Zandler, Amer. J. Phys., 35:12 (1967), 1118-1119.

[3] X. L. Yang, S. H. Guo, F. T. Chan, K. W. Wong, W. Y. Ching, Phys. Rev. A, 43:3 (1991), 1186-1196.

[4] S. H. Guo, X. L. Yang, F. T. Chan, K. W. Wong, W. Y. Ching, Phys. Rev. A, 43:3 (1991), 1197-1205.

[5] R. E. Moss, Amer. J. Phys., 55:5 (1987), 397-311.

[6] M. Robnik, V. G. Romanovski, J. Phys. A: Math. Gen., 36:29 (2003), 7923-7951.

[7] G. Barton, Amer. J. Phys., 51:5 (1983), 420-422.

[8] D. Yafaev, J. Phys. A: Math. Gen., 30:19 (1997), 6981-6992.

[9] Q.-G. Lin, Phys. Lett. A, 260:1-2 (1999), 17-23, arXiv: quant-ph/9910044.

[10] W. Dittrich, Amer. J. Phys., 67:9 (1999), 768-775, arXiv: physics/9902070.

[11] В. В. Пупышев, ТMФ, 186:2 (2016), 252-271.

[12] В. В. Пупышев, ТMФ, 186:1 (2016), 123-141.

[13] В. И. Смирнов, Курс высшей математики, т. 1, Наука, М., 1974.

[14] Б. М. Будак, С. В. Фомин, Кратные интегралы и ряды, Физматлит, М., 2002.

[15] М. В. Федорюк, Асимптотические методы для линейных обыкновенных дифференииальных уравнений, Наука, М., 1983.

[16] Г. Бейтмен, А. Эрдейи, Высшие трансиендентные функиии, т. 1: Гипергеометрическая функиия. Функиии Лежандра, Наука, М., 1973. 
[17] М. Абрамовиц, И. Стиган (ред.), Справочник по специальным функииям с формулами, графиками и математическими таблицами, Наука, М., 1979.

[18] Ю. Люк, Специальные математические фукиии и их аппроксимачии, Мир, М., 1980.

[19] А.П. Прудников, Ю.А. Брычков, О.И. Маричев, Интегралы и ряды. Элементарные функиии, Наука, М., 1981; Интегралы и рлды. Специальные функции, Наука, М., 1983.

[20] C.-E. Fröberg, Rev. Modern Phys., 27:4 (1955), 399-411.

[21] Z. Idziaszek, T. Calarso, P. Zoller, Phys. Rev. A, 76:3 (2007), 033409, 16 pp.

Поступила в редакцию 17.08.2015, после доработки 28.09.2015 